\title{
Chinese Herbal Medicine Suyin Detoxification Granule Inhibits Pyroptosis and Epithelial- Mesenchymal Transition by Downregulating MAVS/NLRP3 to Alleviate Renal Injury
}

\author{
Yiye Zhu',2 \\ Guoshun Huang ${ }^{1,2}$ \\ Yang Yang ${ }^{1,2}$ \\ Chen Yong ${ }^{1,2}$ \\ Xiang $\mathrm{Yu}^{\mathrm{l}, 2}$ \\ Gang Wang ${ }^{3}$ \\ Lan $\mathrm{Yi}^{\mathrm{l}, 4}$ \\ Kun Gao ${ }^{1,4}$ \\ Fang $\operatorname{Tian}^{2}$ \\ Shushu Qian ${ }^{2}$ \\ Enchao Zhou ${ }^{1,4}$ \\ Yanqin Zou ${ }^{1,3,4}$
}

'Department of Nephrology, Jiangsu Province Hospital of Chinese Medicine, Affiliated Hospital of Nanjing University of Chinese Medicine, Nanjing, Jiangsu, People's Republic of China; ${ }^{2}$ No. I Clinical Medical College, Nanjing University of Chinese Medicine, Nanjing, Jiangsu, People's Republic of China;

${ }^{3}$ Zou's Nephrology Medicine Intangible Cultural Heritage Inheritance Studio, Nanjing Boda Nephrology Hospital, Nanjing, Jiangsu, People's Republic of China; ${ }^{4}$ Inheritance Studio of Traditional Chinese Medicine Master Yanqin Zou, Jiangsu Province Hospital of Chinese Medicine, Nanjing, Jiangsu, People's Republic of China
Correspondence: Enchao Zhou;

Yanqin Zou

Tel +86-1385I572603; +86-25-86538830

Fax +86-25-86538830

Email snkII0@।63.com;

fsyy00650@njucm.edu.cn
Purpose: Proteinuria is an independent risk factor of chronic kidney disease (CKD). Albumin-induced tubulointerstitial inflammation and epithelial-mesenchymal transition (EMT) via the activation of NLRP3 inflammasome is a potential therapeutic target for CKD. Suyin Detoxification Granule (SDG) improves proteinuria and postpones renal failure. However, the underlying mechanism is still unknown.

Methods: Firstly, the rat model of renal failure was established using intragastric administration of adenine. Renal function, proteinuria, inflammatory indicators in serum, and renal pathology were assessed, and renal immunohistochemical staining of NLRP3 inflammasomes was performed after intervention with low and high concentrations of SDG. Secondly, the model of renal tubular epithelial HK-2 cells was established using albumin in vitro, and the cell viability, EMT phenotype, and the expression of proteins in the NLRP3 inflammasome signaling pathway were measured after the freezedried powder of Suyin Detoxification Prescription (SDP) and CY-09, which is a selective and direct NLRP3 inhibitor, were co-incubated with albumin. ATP, SOD, mitochondrial membrane potential, and ROS were further measured in vitro, and changes in the mitochondrial function after SDP intervention were observed. The mitochondrial antiviral signaling protein (MAVS) was knocked down using siRNA, and the interaction between MAVS and NLRP3 was verified using Western blotting, polymerase chain reaction (PCR), and immunofluorescence.

Results: SDG improved renal function and proteinuria, alleviated renal fibrosis, and reduced serum inflammation and the expression of the components of the NLRP3 inflammasome in the kidney. In vitro, SDP and CY-09 enhanced cell viability after injury with albumin and inhibited pyroptosis induced by the NLRP3 inflammatory signaling pathway and expression of proteins involved in EMT. It was further found that SDP alleviated the mitochondrial dysfunction caused by albumin. The knockdown of MAVS reduced the expression of NLRP3 pathway proteins and their mRNA levels and also weakened the co-localization of NLRP3, thus, reducing cell pyroptosis.

Conclusion: SDP protected renal tubular epithelial cells from cell pyroptosis and EMT by regulating the albumin-induced mitochondrial dysfunction/ MAVS/ NLRP3-ASC-caspase-1 inflammasome signaling pathway.

Keywords: Suyin Detoxification Granule, renal tubular epithelial cells, mitochondrial dysfunction, MAVS, NLRP3 inflammasome, pyroptosis, EMT 


\section{Introduction}

Chronic kidney disease (CKD) is an increasingly serious public health problem. The prevalence of CKD is $8 \%-16 \%$ worldwide. ${ }^{1}$ Since renal failure is progressive and irreversible and there is a lack of specific drugs to protect renal function, the quality of life of patients with CKD is threatened and it burdens the patients' families and the society economically. ${ }^{2}$ Alleviating renal tissue injury and renal dysfunction is a great challenge faced by clinicians and researchers. ${ }^{3}$

In the process of renal fibrosis, intrinsic renal cells are damaged due to the pathogenic factors, leading to increased collagen deposition, resulting in gradual sclerosis of renal parenchyma, scarring, and a loss of basic metabolic function, causing end-stage kidney disease (ESKD). ${ }^{4}$ Renal fibrosis is characterized by persistent inflammation, an increase in and aggregation of myofibroblasts, and abnormal deposition of extracellular matrix (ECM). There is increasing evidence that epithelialmesenchymal transition (EMT) plays an important role in renal tubulointerstitial fibrosis. ${ }^{5,6}$ During EMT, cell morphology changes, cell migration increases, the adhesion factor E-cadherin and the tight junction protein ZO-1 decrease, and the extracellular matrix proteins, such as Fibronectin and Collagen type I, increase. ${ }^{7}$

Inflammasome research is gaining popularity in recent years. Among the identified inflammasomes, NLRP3 inflammasome is widely studied. ${ }^{8,9}$ NLRP3 inflammasome integrates both infectious and non-infectious tissue injury signals. The most classical pathway is the activation of caspase-1, which induces the secretion of proinflammatory cytokines IL-1 $\beta$ and IL-18 and inflammatory cell death- (pyroptosis), promoting host defense against pathogens and tissue repair. ${ }^{10}$ Available data suggested that in addition to renal mononuclear phagocytes, certain intrinsic kidney cells, such as renal tubular epithelial cells, podocytes, glomerular endothelial cells, and mesangial cells, also contain large amounts of NLRP3. ${ }^{11}$ In several CKD disease models, the release of Il-1 $\beta$ after the activation of NLRP3 inflammasome is associated with renal inflammation, EMT, and fibrosis. ${ }^{12-14}$ Therefore, NLRP3 inflammasome is a potential therapeutic target for CKD.

After more than 2500 years of continuous development, traditional Chinese Medicine (TCM) has gradually become a well-defined treatment system and has been widely used in the treatment of diseases. Suyin
Detoxification Prescription (SDP) promotes blood circulation, eliminates heat, and detoxifies the kidney. SDP has been used in the treatment of chronic renal failure for several years. In our previous study, it was found that SDP's monarch drugs Zi Su Ye [Folium Perillae] and Yin Chen [Herba Artemisiae Scopariae] have antioxidant and anti-apoptotic effects, ${ }^{15}$ but the mechanism underlying SDP's renal protective function is still unclear. Adenine causes renal failure and proteinuria in rodents, which is the model for studying CKD. ${ }^{16}$ We used the adenine rat renal failure model to explore the effects of SDP on urinary proteins, renal function, and inflammatory indicators. In vitro, albumin-induced injury to the human renal tubular epithelial HK-2 cells was used to simulate the destruction of renal tubules using proteinuria, explore the effect of SDP on mitochondrial dysfunction leading to the activation of NLRP3 by MAVS, and study the induction of pyroptosis and EMT in HK-2 cells.

\section{Materials and Methods \\ Reagents}

Adenine (A8626) was purchased from Sigma-Aldrich (MO, USA). Albumin from bovine serum (BSA), Cohn Fraction V (4240GR100) was purchased from BioFROXX (Germany). CY-09 (HY-103666) was purchased from MedChemExpress (NJ, USA). SDG was purchased from the Jiangsu Provincial Hospital of Traditional Chinese Medicine (2005001, Jiangsu, CN). It is currently an inhospital preparation at the Jiangsu Provincial Hospital of Traditional Chinese Medicine and is marketed by the hospital. Antibodies against Collagen Type I (67288-1-lg), Fibronectin (66042-1-lg), and MAVS (14341-1-AP) were purchased from Proteintech (Chicago, USA). The primary antibody against Cleaved IL-1 $\beta$ (AF4008) was purchased from Affinity Biosciences (OH, USA). The primary antibody against pro Caspase- $1+$ p10 + p12 (ab179515) was purchased from Abcam (Cambridge, UK). The primary antibody against Gasdermin D (N terminal; ER1901-37) was purchased from HUABIO (Zhejiang, China). The primary antibody against NLRP3 (NBP2-12446) was purchased from Novus (CO, USA). Mito-Tracker Red CMXRos (C1035) was purchased from Beyotime (Shanghai, CN). HRP-conjugated Goat Anti-Rabbit IgG $(\mathrm{H}+\mathrm{L})$ and HRPconjugated Goat Anti-Mouse IgG $(\mathrm{H}+\mathrm{L})$ were purchased from Servicebio (Hubei, CN). CoraLite488-conjugated Affinipure Goat Anti-Rabbit IgG $(\mathrm{H}+\mathrm{L})$ and CoraLite594- 
conjugated Affinipure Goat Anti-Rabbit $\operatorname{IgG}(\mathrm{H}+\mathrm{L})$ were purchased from Biosharp (Shanghai, CN).

\section{LC-MS/MS Analysis of SDP}

SDP was prepared using Zi Su Ye [Folium Perillae], Yin Chen [Herba Artemisiae Scopariae], Liu Yue Xue [Serissa japonica Thunb.], Tu Fu Lin [Smilax glabra Roxb.], Hong Hua [Carthamus tinctorius L.], Wu Ling Zhi [Faeces Trogopterori], Pu Huang [Typha angustifolia L.], Da Huang [Rheum Officinale], Mu Li [Concha Ostreae], Fu Ling [Poria], Huang Qi [Radix Astragali], and Shan Zhu Yu [Cornus officinalis Sieb]. All the medical herbs were sourced from the Jiangsu Provincial Hospital of TCM. Then, the Suyin Detoxification Lyophilized Powder was prepared at the Institute of the pharmacology at the Nanjing University of Chinese Medicine. Apigenin (B20981), Rhein (B20245), Aloe-emodin (B20772), Formononetin (B20836), and Ferulic acid (B20007) were purchased from Yuanye BioTechnology (Shanghai, China). 4'-hydroxyacetophenone (BCBV5820) was purchased from Sigma-Aldrich (St. Louis, USA). Chlorogenic acid (HY-N0055), Quercetin (HY-18085), and Scoparone (HY-N0228) were purchased from MedChemExpress (NJ, USA). We used liquid chromatography tandem-mass spectrometry (LC-MS/MS) to analyze SDP. After separation on an Xbridge C18 column $(2.1 \times 100 \mathrm{~mm} ; 3.5 \mu \mathrm{m}$; Waters, USA $)$ using an acetonitrilewater gradient system, the peaks were analyzed using mass spectrometry (MS) using ESI ionization in MRM mode. Data were collected and processed using the LC-MS/MSassociated software (Table 1 and Figure S1).

\section{Animals}

Male SD rats ( $\mathrm{n}=24 ; 12$ weeks of age; weight, 200-230 g) were purchased from the Shandong Jinan Yueshi Animal Breeding Center (Shandong, China). After one week of adaptive feeding, adenine was administered to the rats intragastrically. They were randomly divided into four groups ( $\mathrm{n}=6$ per group): control, adenine (Ade), SDG low-dose (SDG-L), and SDG high-dose (SDG-H) groups. In the Ade group, adenine $(2.5 \%, 200 \mathrm{mg} / \mathrm{kg} / \mathrm{d})$ was administered intragastrically every day for 4 weeks and then on alternate days for another 4 weeks to maintain the progression of the disease. The high-dose SDG group was administered with $10 \mathrm{~g} / \mathrm{kg} / \mathrm{d}$ SDG and the low-dose group was administered $5 \mathrm{~g} / \mathrm{kg} / \mathrm{d} \mathrm{SDG}$, and no more than $2 \mathrm{~mL}$ of the compound solution a day was administered from the second week until the end of the experiment.

\section{Ethics Approval}

All animal experiments conformed to the Guidelines for the Care and Use of Laboratory Animals and were approved by the Animal Ethics Committee of the Affiliated Hospital of Nanjing University of Chinese Medicine (permit number: 2020DW-27-02).

\section{Urinary and Serum Sample Analysis}

Blood urea nitrogen, serum creatinine, serum uric acid, serum cystatin, and albumin were measured using an automatic biochemical analyzer (Dimension EXL200, Siemens healthineers, USA). Urinary ACR was detected using a urine analyzer (Uritest-500B, URIT, CN). Serum IL-1, IL-1 $\beta$, IL-18, and MCP-1 were measured using ELISA kits (Meimian, $\mathrm{CN}$ ). All procedures were performed according to the respective manufacturers' instructions.

\section{Renal Pathology}

After the renal tissues were fixed with $10 \%$ formalin, samples were taken, and step-up alcohol dehydration, transparency, immersion, embedding, sectioning, routine $\mathrm{H} \& \mathrm{E}$ staining, and Masson and Pas staining were

Table I Chinese Medicine Simplex Components of SDP Were Measured by LC-MS/MS

\begin{tabular}{|l|c|l|c|c|c|}
\hline Standard & $-/+$ & $\mathbf{m} / \mathbf{z}$ & DP (Volts) & CE(Volts) & Concentration( $\mu \mathrm{g} / \mathbf{g})$ \\
\hline Formononetin & + & $269 \rightarrow 253.7$ & 120 & 28 & 51.18 \\
Scoparone & + & $207.2 \rightarrow|5| .2$ & 82 & 28 & 0.06 \\
Chlorogenic acid & - & $353.1 \rightarrow \mid 90.4$ & -50 & -30 & 11495.85 \\
Apigenin & - & $268.8 \rightarrow \mid 16.6$ & -100 & -50 & 150.16 \\
Rhein & - & $282.8 \rightarrow \mid 82.7$ & -60 & -40 & 6739.17 \\
Aloe-emodin & - & $268.7 \rightarrow 239.7$ & -100 & -30 & 13.86 \\
Ferulic acid & - & $192.7 \rightarrow \mid 33.9$ & -60 & -19 & 1550.10 \\
Quercetin & - & $300.9 \rightarrow \mid 50.6$ & -100 & -28 & 64.03 \\
4'-hydroxyacetophenone & - & $134.7 \rightarrow 92.8$ & -60 & -23 & 86.83 \\
\hline
\end{tabular}


performed. Histopathological changes were observed using a light microscope (Eclipse Ni-U, Nikon, Japan). ImageJ was used to measure the Masson-stained area.

\section{Renal Immunohistochemical Analysis}

After the paraffin sections were dehydrated, they were placed in the box filled with EDTA antigen repair buffer $(\mathrm{pH} 9.0)$ for antigen repair in the microwave oven (medium fire for $8 \mathrm{~min}$ to boil, cease fire for $8 \mathrm{~min}$ and then medium- low fire for $7 \mathrm{~min}$ ). After natural cooling, they were washed with PBS. The sample slides were placed in $3 \%$ hydrogen peroxide solution and incubated for $25 \mathrm{~min}$ at room temperature in dark. After blocking with $3 \%$ bovine serum albumin at room temperature for $30 \mathrm{~min}$, the samples were incubated with a primary antibody (diluted with PBS) overnight at $4{ }^{\circ} \mathrm{C}$. Slides were then washed with PBS and incubated with a secondary antibody HRP-conjugated Goat Anti-Rabbit/Mouse IgG $(\mathrm{H}+\mathrm{L})$ at room temperature for $1 \mathrm{~h}$. After drying, a freshly prepared 3,3'-diaminobenzidine chromogenic reagent (DAB staining fluid, Servicebio, CN) was added to the marked tissue. Slides were observed using a light microscope (Eclipse NiU, Nikon, Japan) until the nuclei turned yellow-brown, and then counterstained with hematoxylin staining solution. Finally, the samples were dehydrated, cleared in xylene for $5 \mathrm{~min}$, and mounted with resin mounting medium. The tissues were observed using a light microscope.

\section{Cell Culture}

The normal human renal tubular epithelial HK-2 cell line was purchased from Cellcook Biotechnology Co, LTD (Guangzhou, China) and the STR gene identification certificate was obtained. The cells were grown in Dulbecco's Modified Eagle's medium/F-12 (DMEM/F12) containing $10 \%$ FBS (GIBCO, USA) in an incubator containing 5\% $\mathrm{CO}_{2}$ at $37^{\circ} \mathrm{C}$.

\section{Assessment of Cell Viability Using the Cell Counting Kit-8}

Cell viability was measured using the CCK-8 kit (Dojindo, China). Cells were incubated in 96-well plates $(10,000$ cells per well) with $100 \mu \mathrm{L}$ culture medium containing $5 \%$ FBS in each well. After stimulation with the drug, the culture medium was removed, and $100 \mu \mathrm{L}$ culture medium containing $10 \% \mathrm{CCK}-8$ detection solution was added to each well. After incubation for $1 \mathrm{~h}$, the absorbance was measured using a spectrometer at $450 \mathrm{~nm}$. Cell viability was expressed as a percentage of the control.

\section{Cell Wound Scratch Assay}

Vertical lines were marked on the outer surface of each well of the 6-well plate. The cells were planted in the 6-well plate with a density of 200,000 cells per well. After the cells were fused to about $90 \%$, the culture medium was removed. A thin "wound" perpendicular to the marker lines was introduced by scratching the cells with a pipette tip. The cells were lightly washed twice with PBS, and each drug was diluted in a serum-free medium and then added to the 6-well plates. After detection, images were captured using a light microscope. ImageJ was used to measure the scratch area.

\section{Western Blotting Analysis}

Sample preparation: After the treatment, the cells were lysed with a solution containing protease and phosphatase inhibitors (Beyotime, CN). After the lysis, the lysate was collected and subjected to ultrasonication and centrifugation. The supernatant was collected, SDS loading buffer was added to it, and the solution was boiled at $100{ }^{\circ} \mathrm{C}$ for $10 \mathrm{~min}$. The samples were then subjected to SDS-PAGE electrophoresis (Bio-RAD, China) on a $10 \%$ or $12.5 \%$ gel and transferred to polyvinylidene fluoride (PVDF) membranes. The membranes were blocked with 5\% non-fat dried milk in PBST (PBS with 1\%o Tween-20) at room temperature for $1 \mathrm{~h}$, incubated with primary antibody at $4{ }^{\circ} \mathrm{C}$ overnight, lightly washed by PBST, incubated with secondary antibody for $1 \mathrm{~h}$, and washed again. The bands were visualized using an imaging system and analyzed using Image Lab software.

\section{Immunocytochemistry}

Cells were fixed with 4\% paraformaldehyde for $20 \mathrm{~min}$ at room temperature and permeabilized with $0.1 \%$ Triton $\mathrm{X}-100$ in PBS. After blocking with 3\% bovine serum albumin in PBS, the samples were incubated with a primary antibody (diluted 1:200) overnight at $4{ }^{\circ} \mathrm{C}$. Then, the samples were incubated with a CoraLite488-conjugated Affinipure Goat Anti-Rabbit IgG $(\mathrm{H}+\mathrm{L})$ (diluted 1:200) for $1 \mathrm{~h}$ at room temperature. After the samples were slightly shaken dry, CY3-TSA (Tyramide signal amplification) was dropped into the circle and incubated for $10 \mathrm{~min}$ at room temperature in dark. Another primary antibody (diluted 1:200) was added and samples were incubated at $4{ }^{\circ} \mathrm{C}$ overnight. After washing with PBS, samples were incubated 
Table 2 Primer Sequences

\begin{tabular}{|l|l|}
\hline $\begin{array}{l}\text { Human-MAVS-S } \\
\text { Human-MAVS-A }\end{array}$ & GCCCATCAACTCAACCCGT \\
\cline { 2 - 2 } & AACGGGCTGTCTACCTGGGAT \\
\hline $\begin{array}{l}\text { Human-NLRP3-S } \\
\text { Human-NLRP3-A }\end{array}$ & ATTCGGAGATTGTGGTTGGG \\
\cline { 2 - 2 } & GAGTCTGGTCAGGGAATGGCT \\
\hline $\begin{array}{l}\text { Human- } \beta \text {-actin-S } \\
\text { Human- } \beta \text {-actin-A }\end{array}$ & CTCCTTAATGTCACGCACGAT \\
\cline { 2 - 2 } & CTCCTTAATGTCACGCACGAT \\
\hline
\end{tabular}

with CoraLite594-conjugated Goat Anti-Rabbit IgG $(\mathrm{H}+\mathrm{L})$ (diluted 1:200) for $1 \mathrm{~h}$ at room temperature. After washing with PBS, samples were incubated with TSA for $10 \mathrm{~min}$ at room temperature and stained with DAPI staining solution for $15 \mathrm{~min}$. An anti-fluorescence quenching agent was added. A fluorescence microscope was used to observe the cells using different channels. ImageJ was used to measure the relative fluorescence intensities.

\section{RT-PCR Analysis}

RNA was extracted using the one-step RNA extraction method (Vazyme, CN), and cDNA was synthesized using Direct RT Mix according to the manufacturer's instructions. cDNA was detected and amplified using Direct qPCR MIX-SYBR. The primers used are listed below (Table 2). 7500Fast system was used to detect the mRNA expression and the results were calculated using the $\Delta \Delta \mathrm{CT}$ method. The mRNA levels are expressed as a fraction of those of the control group.

\section{Flow Cytometry Analysis}

Mitochondrial membrane potential was detected using the JC-1 kit (Multi Sciences, Hangzhou, China). After stimulation with drugs, samples were washed with PBS slightly, then trypsin without EDTA was added and samples were incubated at room temperature for 10 min. Then the cells were collected and centrifuged. The supernatant was discarded and the cells were resuspended in a Buffer containing $2 \mu \mathrm{M} \mathrm{JC}-1$. The samples were incubated in a $5 \% \mathrm{CO}_{2}$ incubator at $37^{\circ} \mathrm{C}$ for 20 min. Flow cytometry was performed using an excitation wavelength of $488 \mathrm{~nm}$ and the samples were analyzed using FITC and R-PE channels. Annexin V-FITC/ PI apoptosis kit (Multi Sciences, China) was used to measure cell pyroptosis. After collection, cells in each sample were incubated with $5 \mu \mathrm{L}$ FITC and $2 \mu \mathrm{L}$ PI. Annexin V-FITC and PI detection channels were used to measure cell pyroptosis.

\section{Transient Transfection of Cells with siRNA}

HK-2 cells were transiently transfected with siRNA specifically targeting MAVS. siMAVS and sicontrol (negative reference) were provided by Nanjing KeyGen biological co., LTD and were diluted in Opti medium to a final concentration of $80 \mathrm{nM}$. The transfection reagent used was Lipo 3000 (Thermo Fisher, USA). siRNA and the transfection reagent were mixed, incubated for $15 \mathrm{~min}$, and then added to the cells. After incubation for $8 \mathrm{~h}$, the medium was removed. Transfected cells were then exposed to different stimuli.

\section{Mitochondrial Function Analysis: ATP}

After lysing the cells, $20 \mu \mathrm{L}$ of the supernatant was collected in an opaque 96-well plate. Then, the ATP detection reagent was added to it immediately. A fluorescence microplate reader was used to measure the fluorescence, and the ATP and protein concentrations were calculated. The ATP concentration was converted to $\mathrm{nmol} / \mathrm{mg}$. SOD: The supernatant of the cell lysate was used to measure the level of SOD. After the experiment was performed according to the manufacturer's instructions, the OD value was detected using a spectrometer at a wavelength of $450 \mathrm{~nm}$. The SOD activity and protein concentration were calculated according to the instructions (S0101M, beyotime, Shanghai, CN), and the SOD activity was converted to U/mg. ROS detection: After drug administration, $500 \mu \mathrm{L}$ DCFH-DA fluorescein, at a final concentration of $10 \mu \mathrm{M}$, was added to each well according to the instructions (S0033M, Beyotime, CN), and incubated at $37^{\circ} \mathrm{C}$ for 30 min. After sealing the samples with the anti-fluorescence quenching solution, the samples were directly observed using a forward fluorescence microscope, and images were captured. The relative optical density was measured using ImageJ.

\section{Statistical Analysis}

Values are expressed as means \pm standard deviation. Before performing further statistical analyses, the Shapiro-Wilk test was used to check whether the data were normally distributed. Comparisons between two populations were made using the Student's $t$-test. For comparisons across multiple samples, a one-way analysis of variance followed by Dunnett's test was performed. All analyses were performed using SPSS Statistics 23.0 
software. A $P$-value of less than 0.05 was considered to indicate a statistically significant difference.

\section{Results}

SDG Improved Renal Tissue Fibrosis in Rats with Adenine Nephropathy

Adenine nephropathy rat model did not only cause renal failure but also high proteinuria, which is a rat model of chronic renal failure. Gross examination showed that compared to the control group, the adenine group showed a decrease in body weight, an increase in the renal weight, and a marked swelling, white color, and a granular surface of the kidney, and these effects were attenuated in both the low- and high-dose SDG groups (Figure 1A and B). Renal immunohistochemistry results (Figure 1C) showed that fibrotic markers Collagen I and
A

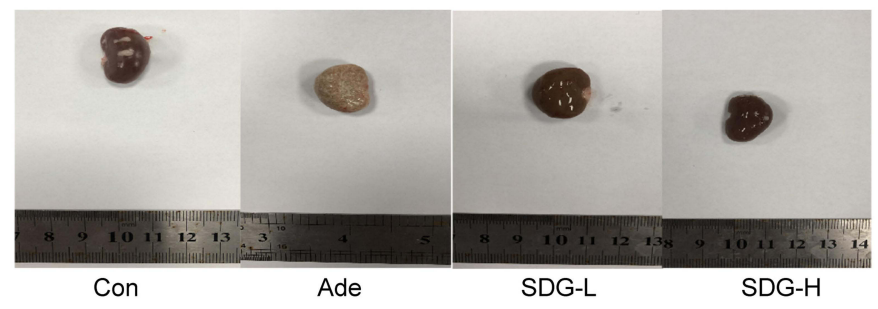

B
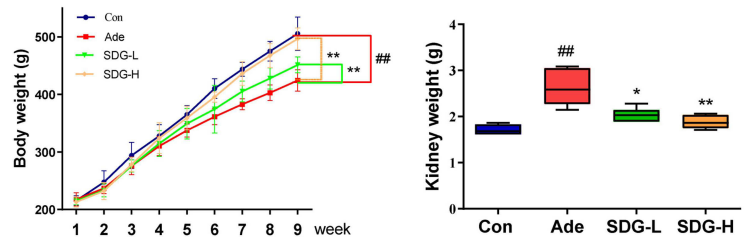

C
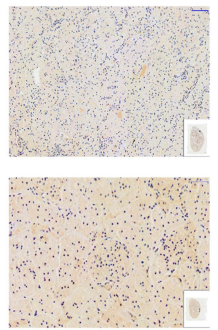

Con

D
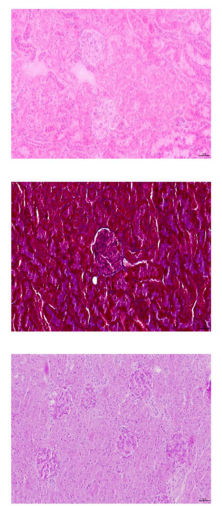

Con

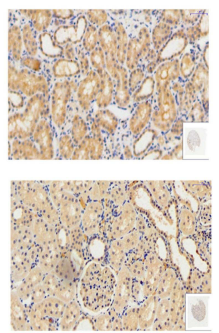

Ade
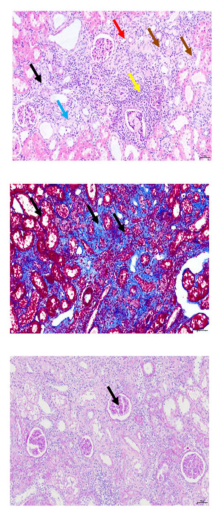

Ade

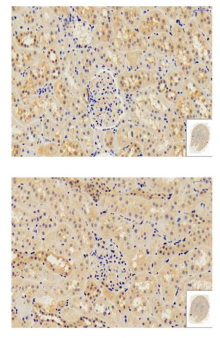

SDG-L
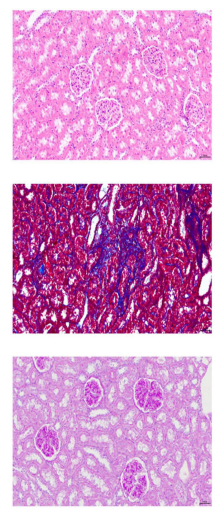

SDG-L

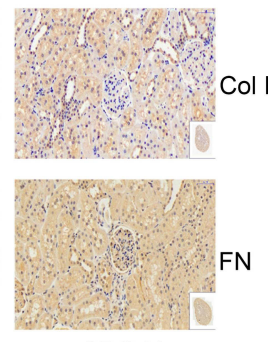

SDG-H
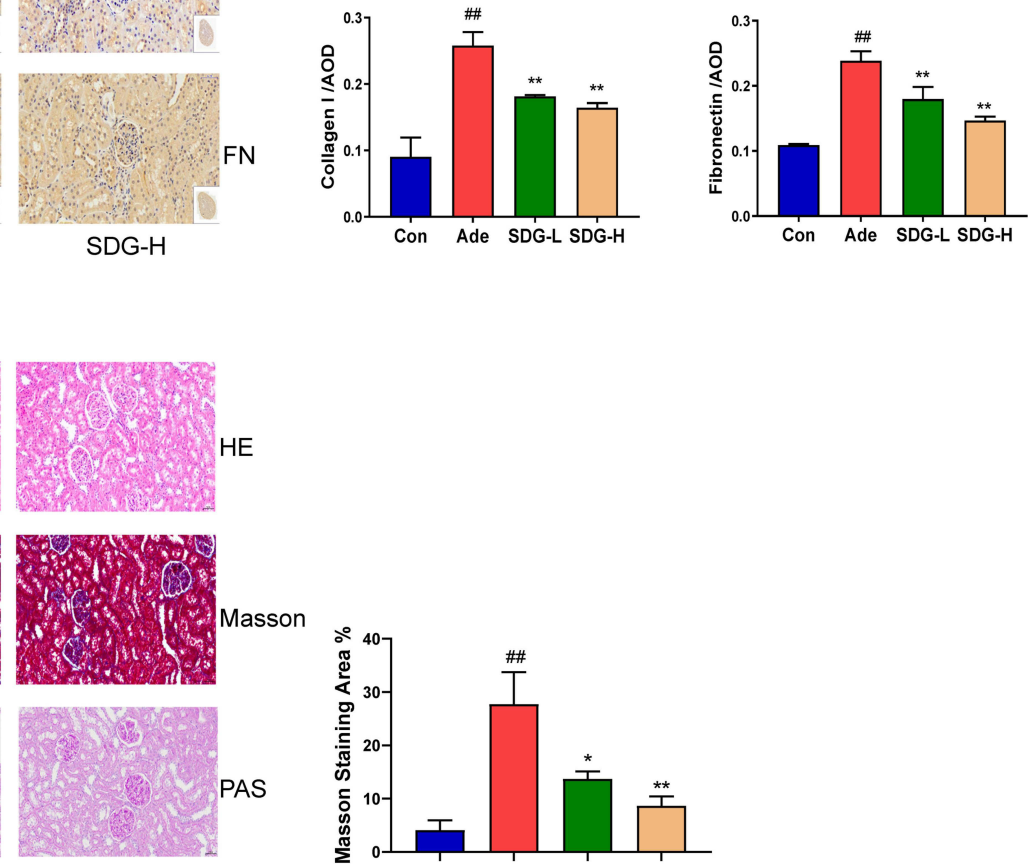

SDG-H

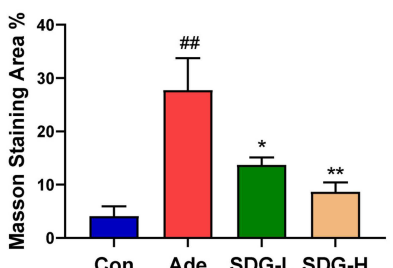

Figure I SDG Improved Renal Tissue Fibrosis in Rats With Adenine Nephropathy. Experimental schedule: The adenine, SDG-L, and SDG-H groups underwent intragastric administration of adenine $(2.5 \%, 200 \mathrm{mg} / \mathrm{kg} / \mathrm{d})$ every day for 4 weeks and then on alternate days for another 4 weeks. SDG-L and SDG-H groups were administered $5 \mathrm{~g} / \mathrm{kg} / \mathrm{d}$ or $10 \mathrm{~g} / \mathrm{kg} / \mathrm{d}$ SDG by gavage once a day. The rats were sacrificed in week 9 . At this point, (A) morphology of the kidneys was observed, (B) the body weight and kidney weight was measured, (C) and representative images of the sections of the kidney were assessed using immunohistology using Collagen I (Col I) and Fibronectin (FN) antibodies. Scale bar $=50 \mu \mathrm{m}$. (D) Hematoxylin and Eosin staining: In the adenine group, a large area of renal interstitial inflammation was observed (brown arrow). The number of renal tubules decreased (black arrow), and kidney atrophy was observed along with a change in their structure (blue arrow). More lymphocyte infiltration was observed in the interstitium (yellow arrow), and the lumen of some renal tubules around the lesion was enlarged; also, the epithelial cells were swollen (red arrow). Masson staining: In the adenine group, there was extensive damage to the cortex, and more collagen fibers were observed at the damaged site and the dark-blue colored regions surrounding the renal tubular interstitium (black arrow). Periodic Acid-Schiff staining: There was no obvious increase in the mesangial matrix and thickening of basement membrane in the glomeruli in the adenine group. However, a small number of renal tubules in the medulla were PAS-positive (black arrow). Scale bar $=50 \mu m$. Data are presented as means $\pm \mathrm{SD} ; \mathrm{n}=6 .{ }^{\#} \mathrm{P}<0.01$ vs control. $* \mathrm{p}<0.05 ; * * p<0.01$ vs the adenine group. 
Fibronectin significantly increased in the Adenine group, and their levels reduced in SDG low- and high-dose groups. H\&E staining of the renal cortex showed that compared to the control group, inflammation was observed in a large part of the renal interstitium in the Adenine group, with a reduced number of renal tubules, atrophy and disappearance of the structure of the kidney, more lymphocyte infiltration in the interstitium, an enlarged lumen of some renal tubules around the lesion, and edema of the epithelial cells. Masson-stained tissue showed high cortical damage, and there was more collagen fiber hyperplasia in the damaged site and the surrounding renal tubule-interstitium, showing a dark blue color. There was no obvious increase in the mesangial matrix and thickening of the basement membrane in glomeruli, observed with PAS staining. However, a small number of renal tubules in the medulla were uniformly positive for PAS, and these lesions were ameliorated in the low- and high-dose SDG groups (Figure 1D). These results suggested that SDG alleviated adenine-induced renal damage.

\section{SDG Alleviated Adenine-Induced Renal Dysfunction and Inflammation in Rats}

As shown in Figure 2A and B, after the treatment with adenine, rat blood urea nitrogen, serum creatinine, serum cystatin, and blood uric acid increased, and the urinary albumin excretion rate and serum albumin decreased. Low- and high-dose SDG groups showed an improvement in the indices of renal function and hypoalbuminemia and a reduction in urinary protein excretion. Serum inflammatory markers IL-1, IL-1 $\beta$, IL-18, and MCP-1 significantly increased in the Adenine group compared to the control group, and the inflammatory response improved in the low- and high-dose SDG groups (Figure 2C). The NLRP3/ caspase-1 /IL-1 $\beta$ signaling pathway, which regulates inflammation through the cleavage and maturation of pro-inflammatory cytokines, has been demonstrated to play a role in regulating inflammation in the kidneys. As shown in Figure 2D, adenine increased NLRP3, caspase-1, GSDMD, and IL-1 $\beta$ in the kidney tissue, and low and high SDG doses decreased their levels. These data suggested that SDG ameliorated adenine-induced renal dysfunction, proteinuria, hypoproteinemia, and inflammatory response, and inhibited the expression of NLRP3, caspase-1, GSDMD, and IL-1 $1 \beta$ in the kidney.

\section{Albumin Induced Renal Tubular Epithelial Cell Injury in vitro}

A growing number of studies suggested that proteinuria is an independent risk factor of renal failure. To study the effect of excessive albumin on the kidney, especially the function of the renal tubule, we used serum albumin to treat the renal tubular epithelial cells and establish the injury model. As shown in Figure 3A, with the increase in the concentration of BSA and the the intervention time, cell viability was affected. Compared to the control group, the low-dose group of $5 \mathrm{~g} / \mathrm{L}$ SDP showed no difference at 12 and 48 $\mathrm{h}$ but showed an increase at $24 \mathrm{~h}$, while the medium- and high-dose groups of $10 \mathrm{~g} / \mathrm{L}$ and $20 \mathrm{~g} / \mathrm{L}$ SDP, respectively, showed a loss of cell activity at 24 and $48 \mathrm{~h}$. Scratch assay results showed that BSA, at $5 \mathrm{~g} / \mathrm{L}, 10 \mathrm{~g} / \mathrm{L}$, and $20 \mathrm{~g} / \mathrm{L}$, improved cell migration at 12 and $24 \mathrm{~h}$. Using light microscopy, it was observed that $24 \mathrm{~h}$ after the intervention, the cell morphology in the BSA group changed from elliptical to a spindle shape, and the volume of the cells increased. A concentration of $10 \mathrm{~g} / \mathrm{L}$ BSA showed the most significant changes (Figure 3B and C). The Western blotting results (Figure 3D) showed that the Vimentin, Collagen I and Fibronectin protein levels increased and the E-cadherin protein level decreased in BSA $10 \mathrm{~g} / \mathrm{L}$ group. To verify whether albumin causes pyroptosis of the renal tubular epithelial cells, the protein levels of NLRP3, ASC, activated caspase-1, GSDMD-N, and activated IL-1 $\beta$ were measured and they were found to be increased after the treatment with BSA at different concentrations (Figure 3E). The most significant increase was found in the group administered with $10 \mathrm{~g} / \mathrm{L}$ BSA. The Western blotting assay showed that along with the increase in the levels of activated caspase- 1 and IL-1 $\beta$, the levels of unactivated precursors of caspase- 1 and IL- $1 \beta$ also increased. These results suggested that albumin can be used to establish renal tubular damage models with decreased functionality, enhanced migration ability, and increased expression of fibrotic marker proteins and NLRP3 series of pyroptosis-related proteins.

\section{SDP May Ameliorate BSA-Induced Cell Injury by Inhibiting the NLRP3/ASC/ Caspase-I Signaling Pathway}

Based on the aforementioned results, we identified $10 \mathrm{~g} / \mathrm{L}$ BSA and a 24-h intervention time as the optimum conditions to establish the cell damage model. To investigate the relationship between NLRP3 inflammasome and EMT, an NLRP3 inhibitor CY-09 was used. CY-09 is a selective and 


\section{A}
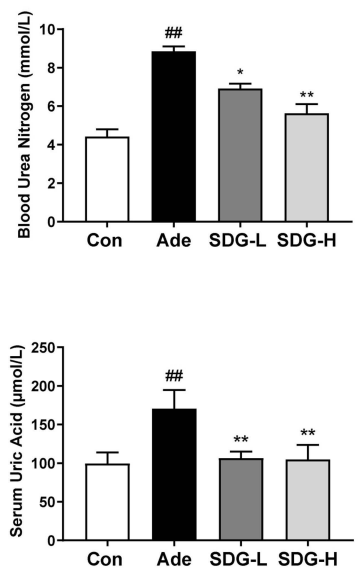

B

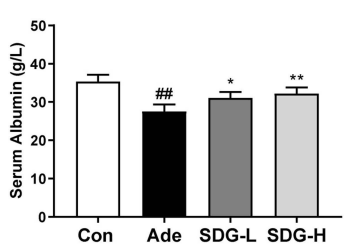

\section{C}
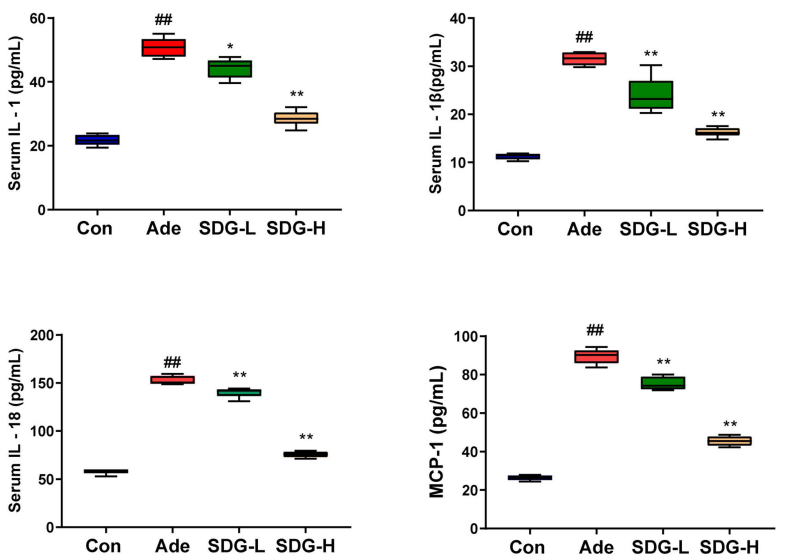
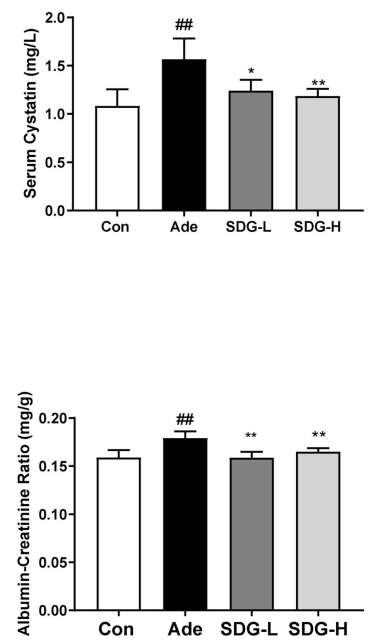

D

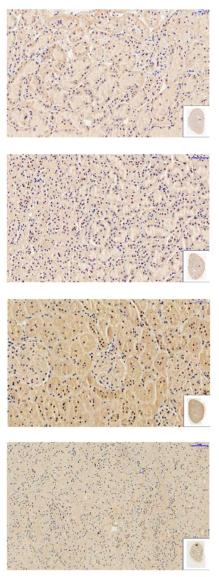

Con
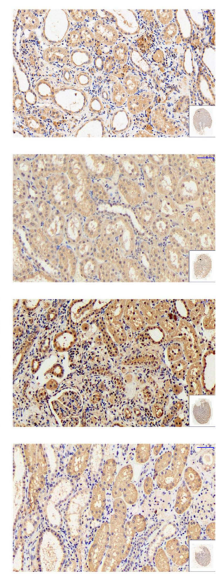

Ade

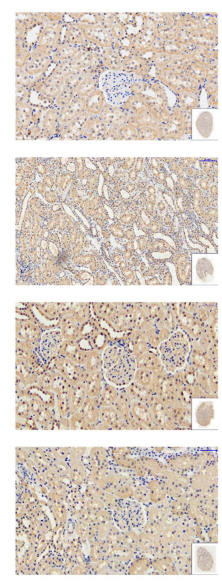

SDG-L

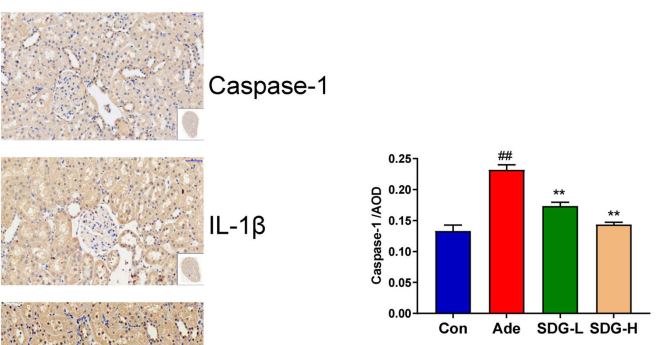

GSDMD

NLRP3

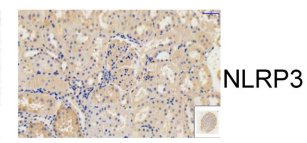

SDG-H

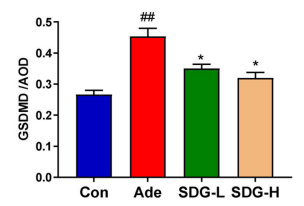

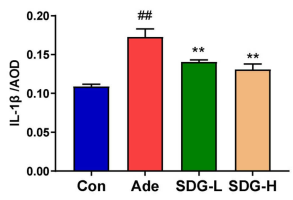

Figure 2 SDG alleviated adenine-induced decrease in the renal function and inflammation in rats. (A) Blood urea nitrogen (BUN), Serum creatinine (Scr), Serum cystatin, Serum uric acid, (B) Serum albumin, Urinary albumin-to-creatinine ratio (ACR), and (C) Serum IL-I, IL-I $\beta$, IL-I8, and MCP-I levels were measured. (D) Representative immunohistology images of the sections highlighting NLRP3, Caspase-I, Gasdermin D (GSDMD), and IL-I $\beta$ using their respective antibodies. Scale bar $=50 \mu$ m. Data are presented as means $\pm S D ; n=6$. ${ }^{\#} P<0.01$ vs control. $* P<0.05 ; * * P<0.01$ vs the adenine group.

direct NLRP3 inhibitor. It directly binds to the ATPbinding motif of the NLRP3 NACHT domain and inhibits NLRP3 ATPase activity, resulting in the suppression of NLRP3 inflammasome assembly and activation. To investigate the protective effect of SDP, using the BSA-induced renal damage model, CY-09 $(5 \mu \mathrm{M})$ was co-incubated with
BSA and SDP $(0.25,0.5$, and $1 \mathrm{~g} / \mathrm{L})$. The results showed that SDP $(1 \mathrm{~g} / \mathrm{L})$ and CY-09 improved cell viability. CY-09 and SDP $(0.5$ and $1 \mathrm{~g} / \mathrm{L})$ improved the cell morphology and abnormal cell migration caused by BSA. The effect of $1 \mathrm{~g} / \mathrm{L}$ SDP was more pronounced (Figure $4 \mathrm{~A}-\mathrm{C}$ ). A concentration of $1 \mathrm{~g} / \mathrm{L}$ SDP was identified as the 
A

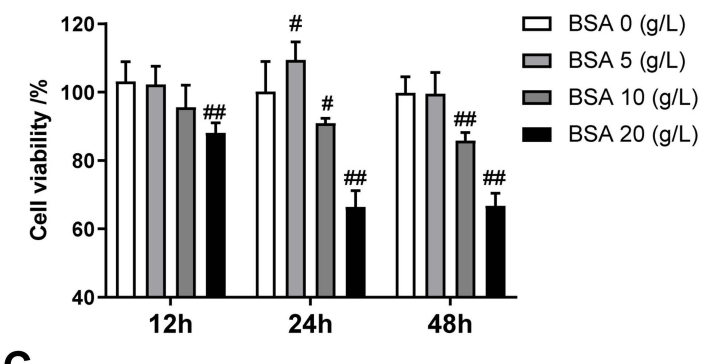

C
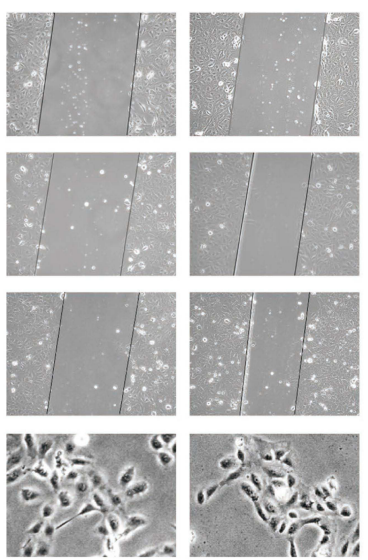

0

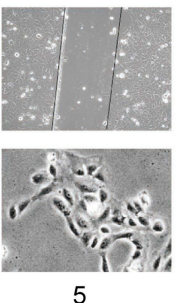

5
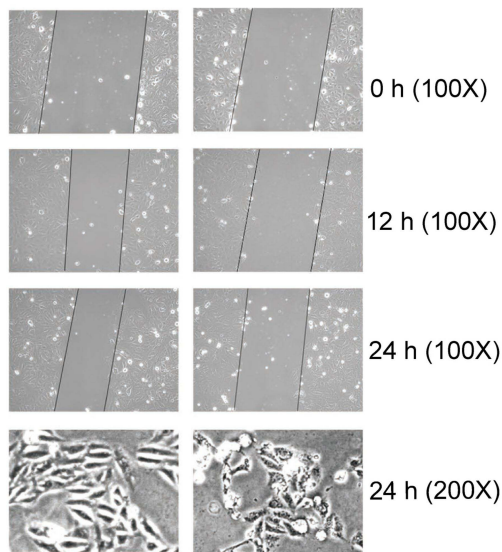

10

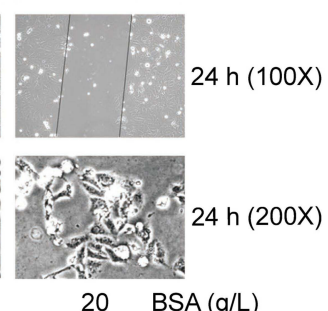

$20 \mathrm{BSA}(\mathrm{g} / \mathrm{L})$
B

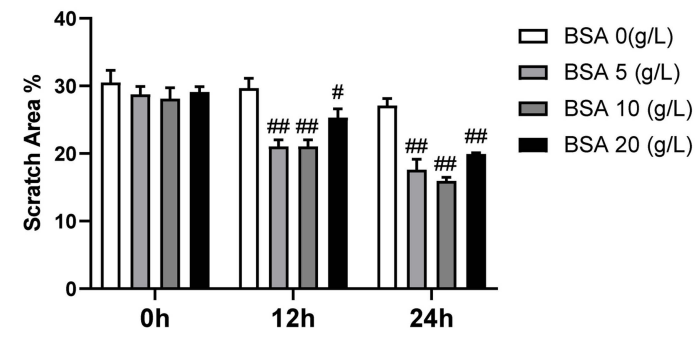

D
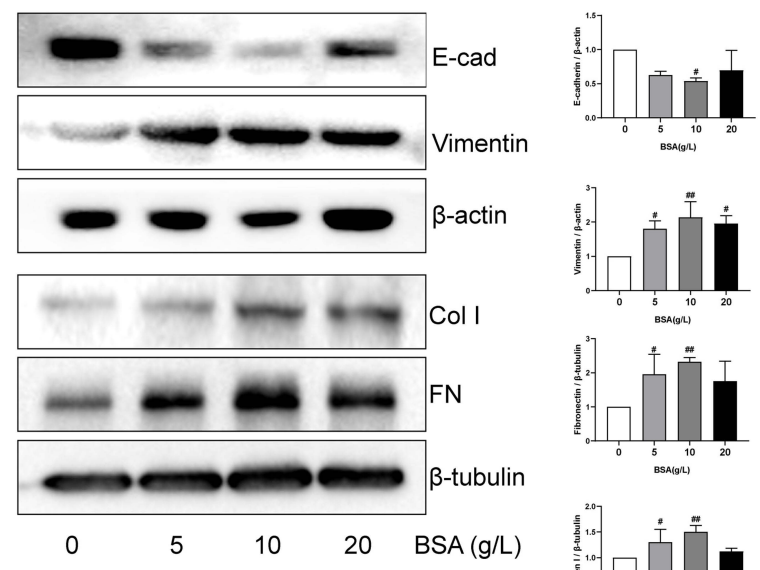

皇

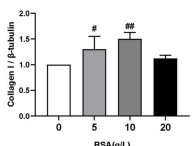

E

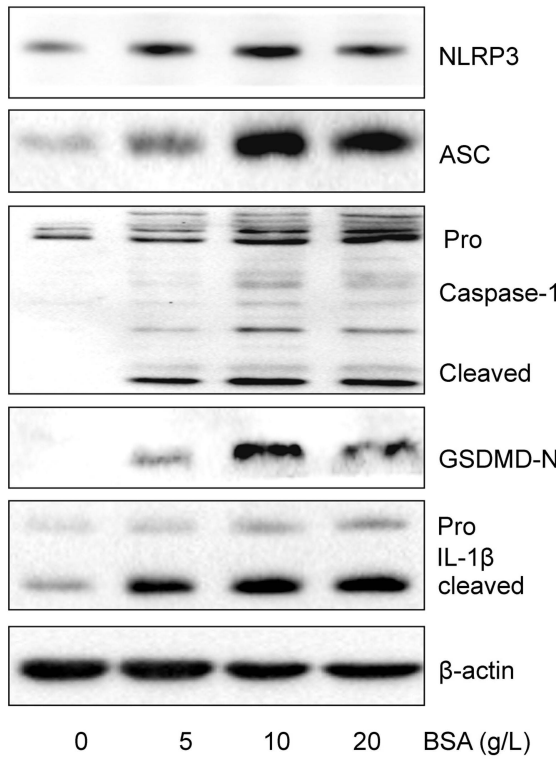

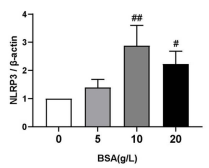
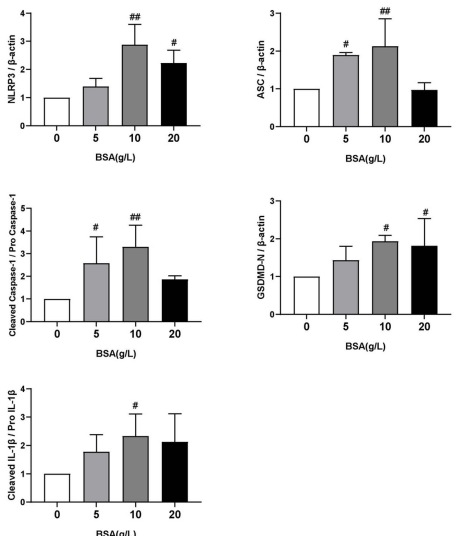

Figure 3 Albumin induced renal tubular epithelial cell injury in vitro. (A) The effect of BSA on cell viability. HK-2 cells in 96-well plates were exposed to the different concentrations $(0,5,10$, and $20 \mathrm{~g} / \mathrm{L})$ of BSA for 12,24 , and $48 \mathrm{~h}$. Cell viability was evaluated using CCK-8 assay. (B, C) The effect of BSA on the cell migration capacity and associated morphological changes. Cell morphology and scratch assays were performed using phase-contrast microscopy (magnification, I00X). (D) The effect of BSA on Vimentin, E-cadherin (E-cad), Fibronectin (FN) and Collagen type I (Col I) protein expression. After incubation for 24 h, cell lysates were subjected to Western blotting to measure Vimentin, E-cadherin, Fibronectin and Collagen type I levels. (E) The effect of BSA on NLRP3 inflammasome proteins. After incubation for 24 h, cell lysates were subjected to Western blotting to measure NLRP3, ASC, Pro Caspase-I with cleaved caspase -I levels, N-terminal of Gasdermin D (GSDMD-N), and Pro IL-I $\beta$ with cleaved IL-I $\beta$. Data are expressed as means $\pm S D ; n=3 .{ }^{\#} p<0.05 ;{ }^{\#} p<0.0$ I vs control. 
A

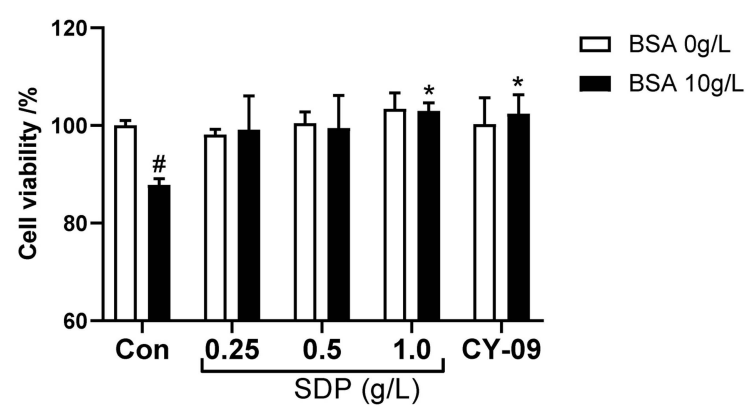

B

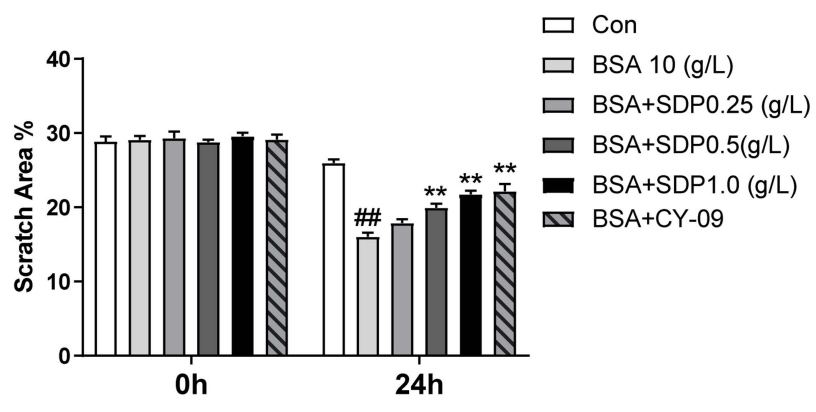

C
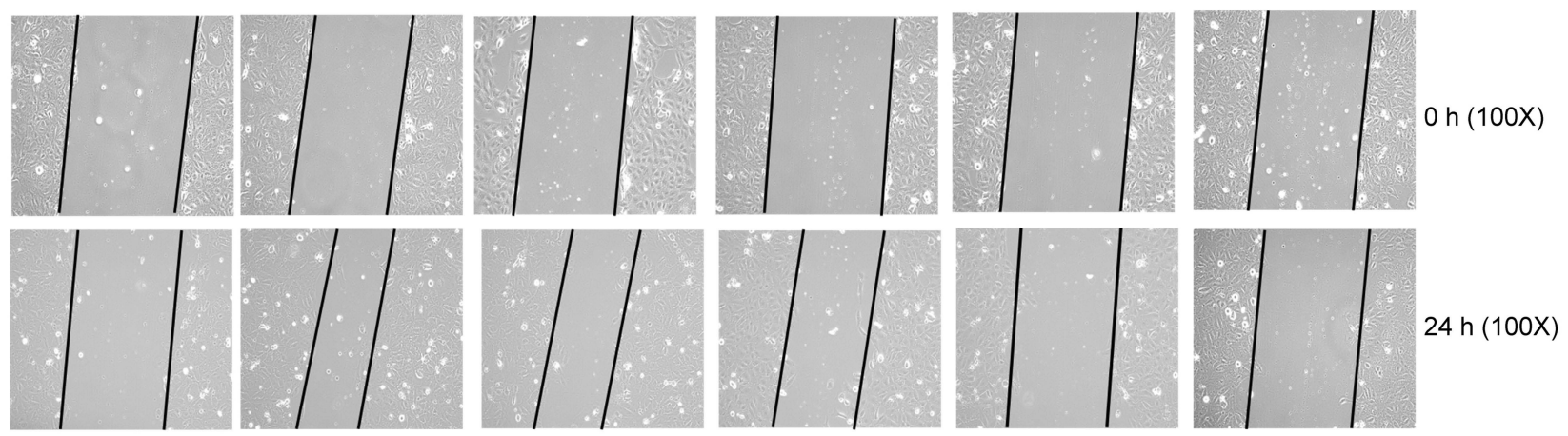

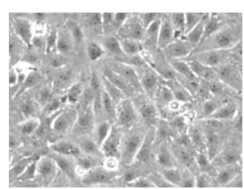

Con

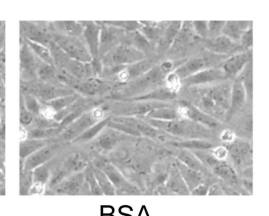

BSA

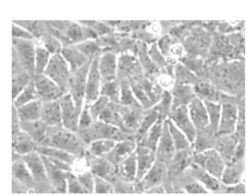

BSA+SDP $0.25 \mathrm{~g} / \mathrm{L}$

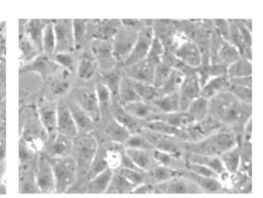

$B S A+S D P 0.5 \mathrm{~g} / \mathrm{L}$

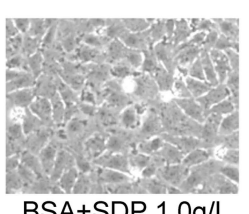

$\mathrm{BSA}+\mathrm{SDP} 1.0 \mathrm{~g} / \mathrm{L}$

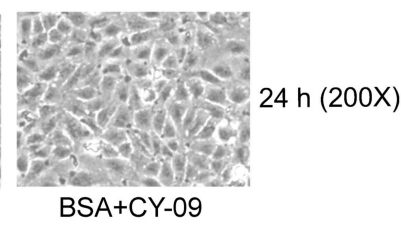

D
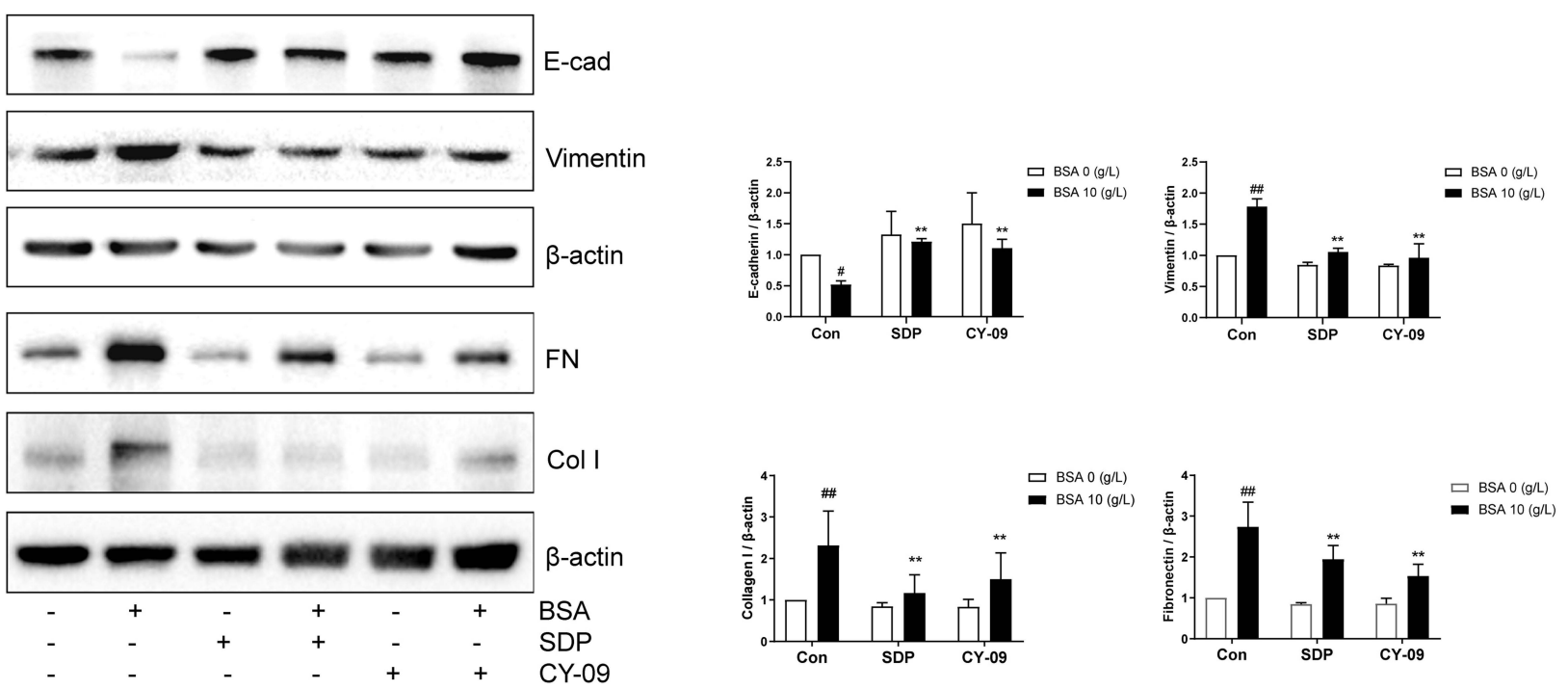

Figure 4 SDP may ameliorate BSA-induced cell injury by inhibiting NLRP3/ASC/ caspase-I signaling pathway. (A) The effects of SDP and CY-09 on BSA-induced cell viability. HK-2 cells in 96 -well plates were co-incubated with SDP $(0.25,0.5$, and $1.0 \mathrm{~g} / \mathrm{L})$ or CY-09 $(5 \mu \mathrm{mol} / \mathrm{L})$ and BSA (10 g/L) for 24 h. Cell viability was evaluated using the CCK-8 assay. (B, C) The effects of SDP and CY-09 on the cell migration capacity and morphological changes induced by BSA. Cell morphology and scratch assay were analyzed using phase-contrast microscopy (magnification, I00X). (D) The effects of SDP and CY-09 on E-cadherin, Vimentin, Fibronectin and Collagen type I protein expression. HK-2 cells were divided into 6 groups: Control, BSA ( $10 \mathrm{~g} / \mathrm{L})$, SDP (I g/L), BSA (10 g/L) + SDP (I g/L), CY-09 $(5 \mu \mathrm{mol} / \mathrm{L})$, and BSA ( $10 \mathrm{~g} / \mathrm{L})+\mathrm{CY}-09(5 \mu \mathrm{mol} / \mathrm{L})$. After incubation for $24 \mathrm{~h}$, cell lysates were subjected to Western blotting to measure E-cadherin, Vimentin, Fibronectin and Collagen type I protein levels. Data are presented as means \pm SD; $\mathrm{n}=3$. ${ }^{\#} P<0.05 ;{ }^{\#} P<0.01$ vs control. $* P<0.05 ; * * P<0.01$ vs BSA group. 
optimum concentration. Western blotting results showed that SDP and CY-09 reduced the protein levels of Vimentin, Collagen I and Fibronectin, and increased the protein level of E-cadherin (Figure 4D). As shown in Figure 5A-C, SDP and CY-09 reduced the expression of NLRP3, thereby inhibiting the activation of the NLRP3ASC-Caspase-1 pyroptosis signaling pathway and reducing the activation of Gasdermin D and the release of IL-1 $\beta$ (Figure 5). However, compared with SDP or CY09, SDP combined with CY-09 seemed to show no difference in this BSA-induced EMT and pyroptosis cell model
(Figure S2). These data suggested that SDP may ameliorate renal tubular EMT by inhibiting NLRP3-mediated pyroptosis.

\section{SDP Ameliorated BSA-Induced \\ Mitochondrial Dysfunction}

Mitochondria not only produce the energy required by the cells but also participate in cellular regulatory processes. Recently, it was shown that they also play a role in regulating immunity. Previous studies have confirmed that mitochondrial dysfunction is one of the processes
A
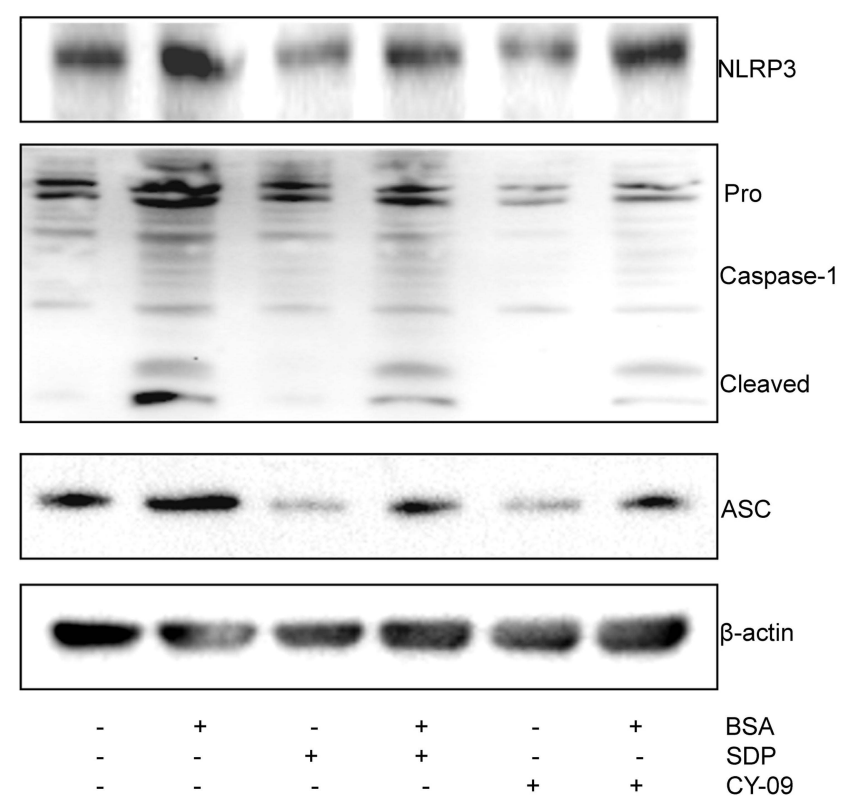

B
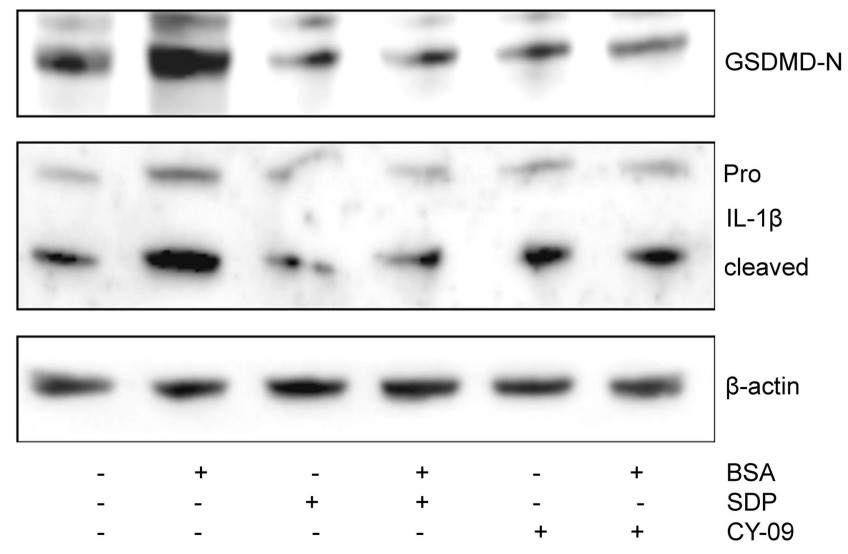

C
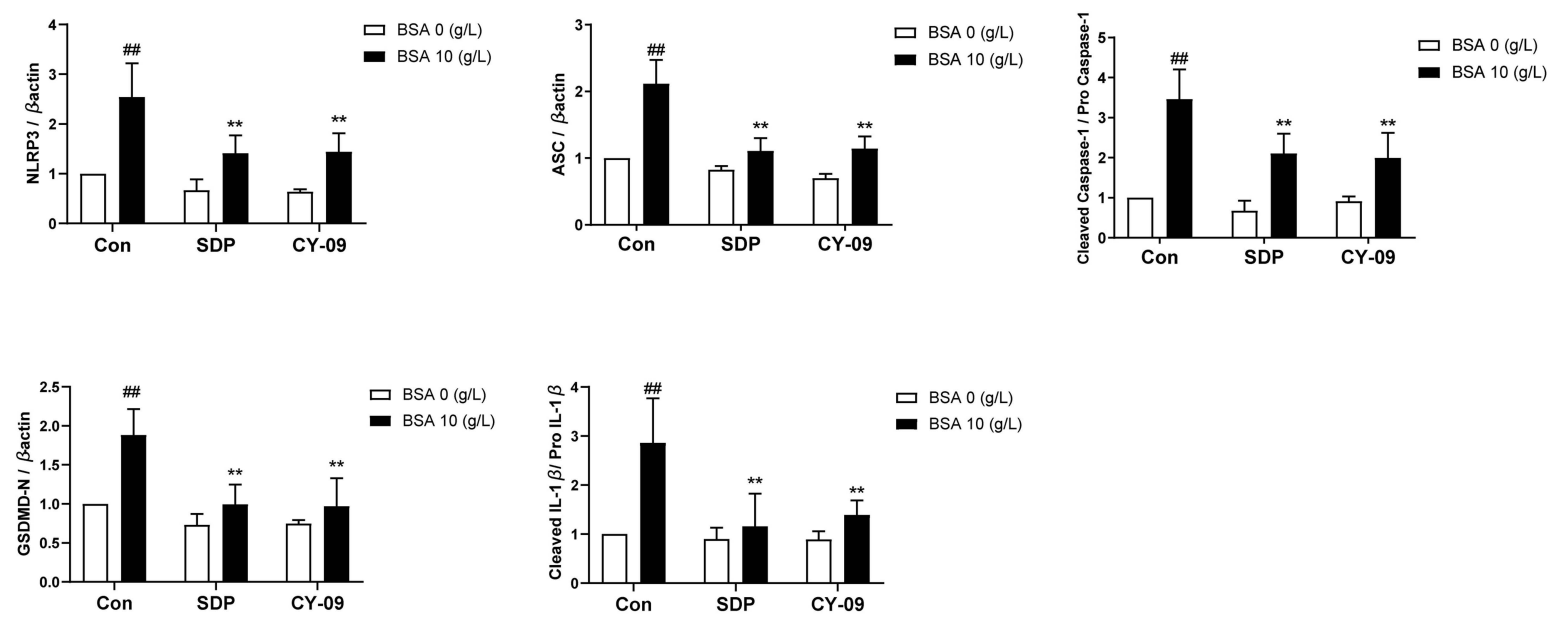

Figure 5 SDP may ameliorate BSA-induced cell injury by inhibiting NLRP3/ASC/ caspase-I signaling pathway. (A-C) The effects of SDP and CY-09 on NLRP3 inflammasome protein expression. After incubation for $24 \mathrm{~h}$, cell lysates were subjected to Western blotting to measure NLRP3, ASC, Pro Caspase-I with cleaved caspase -I, N-terminal of Gasdermin D(GSDMD-N), and Pro IL-I $\beta$ with cleaved IL-I $\beta$ Data are presented as means \pm SD; $n=3$. ${ }^{\#} P<0.01$ vs control. ${ }^{* * P}<0.01$ vs BSA group. 
that lead to the activation of NLRP3. As shown in Figure 6A-E, compared to the BSA group, the SDP group showed a reduction in the release of ATP and the generation of ROS and an increase in the mitochondrial membrane potential and the release of SOD. These results may suggest that SDP could reduce NLRP3 inflammasome activation by alleviating mitochondrial dysfunction.

\section{A}

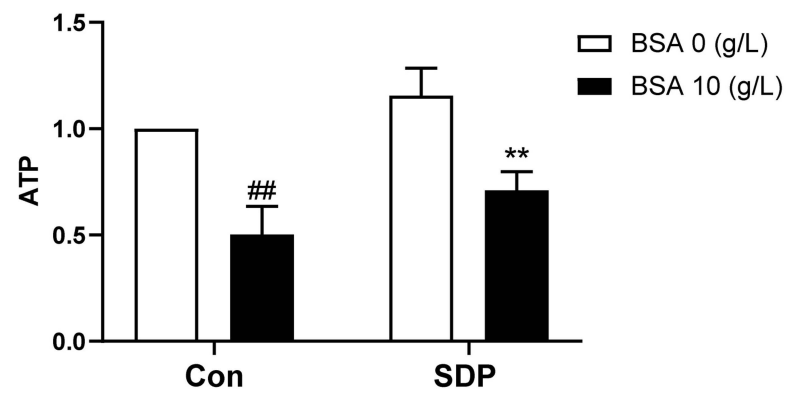

C

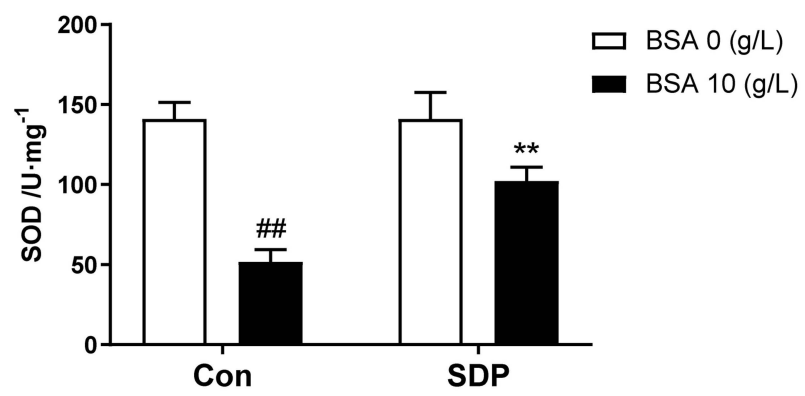

SDP Inhibited the Localization and Activation of NLRP3 in Mitochondria by Regulating MAVS Protein

MAVS, localized to the outer membrane of mitochondria, promotes the localization of NLRP 3 to the mitochondria and induces their activation. As shown in Figure 7A, the expression of NLRP3 also decreased after the MAVS gene

B

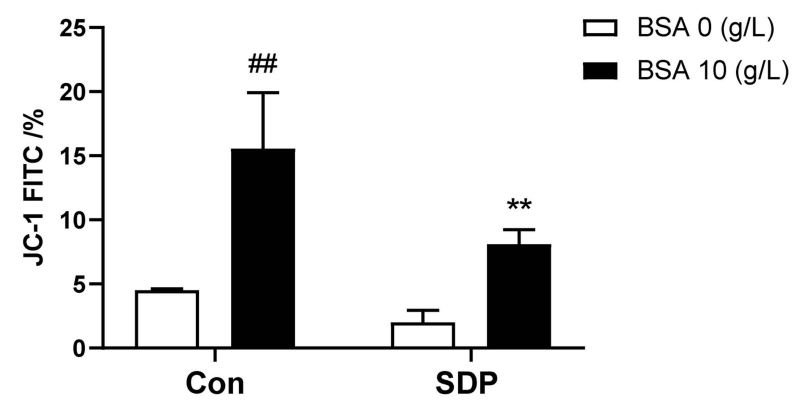

D

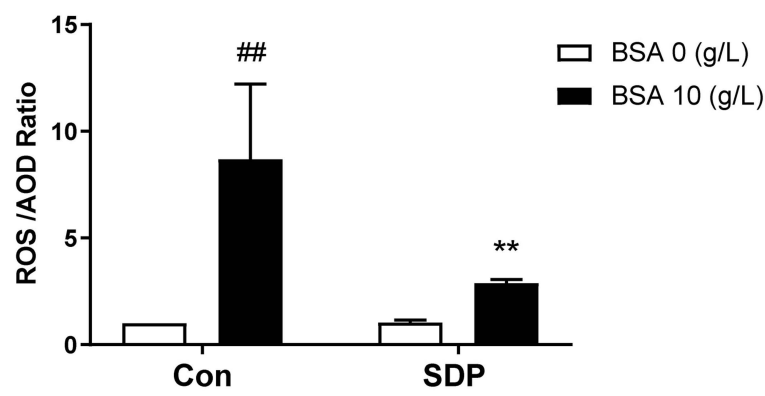

$\mathbf{E}$

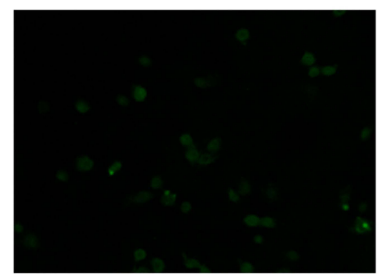

-

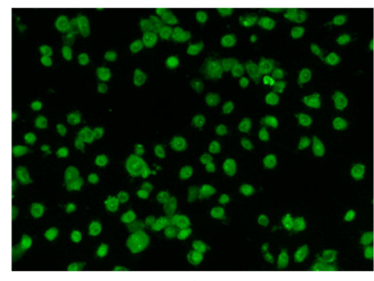

$+$
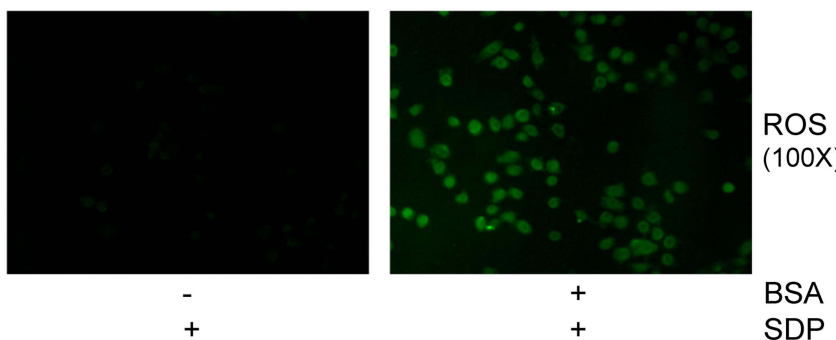

Figure 6 SDP ameliorated BSA-induced mitochondrial dysfunction. (A) The effect of SDP on BSA-induced cell adenosine-triphosphate (ATP) release. After incubation for $24 \mathrm{~h}$, cell lysates were subjected to ATP assay using the kit. A fluorescence microplate reader was used to measure the ATP in the samples. (B) The effect of SDP on BSAinduced changes in mitochondrial membrane potential (MMP). JC-I assay kit was used to measure the MMP. FITC channel was used to calculate cells with mitochondrial damage. (C) The effect of SDP on BSA-induced superoxide dismutase (SOD) release. A SOD assay kit was used to measure the released SOD. (D, E) The effect of SDP on BSA-induced production of reactive oxygen species (ROS). The corresponding kit was used to measure ROS. The cells were observed using fluorescence microscopy (magnification, I00X). Data are expressed as means $\pm \mathrm{SD} ; \mathrm{n}=3$. \#\#, $\mathrm{P}<0.0 \mathrm{I}$ vs control. $* * \mathrm{P}<0.0 \mathrm{I}$ vs BSA group. 
A
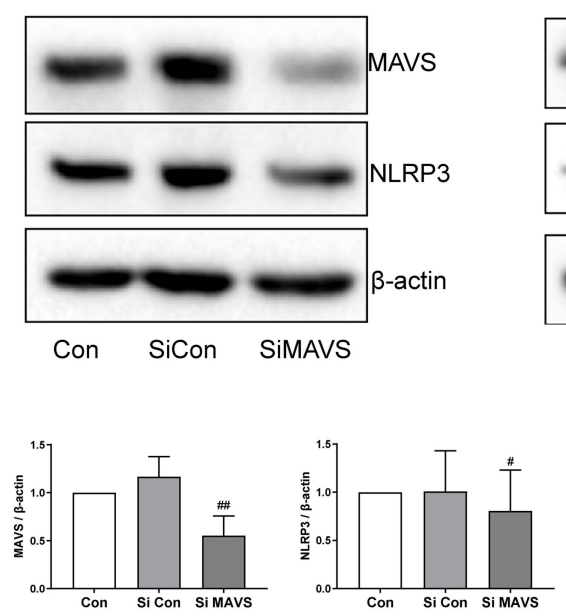

D

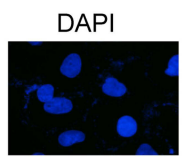

Mito-Tracker

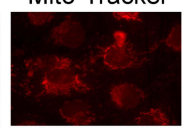

DAPI

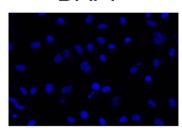

MAVS
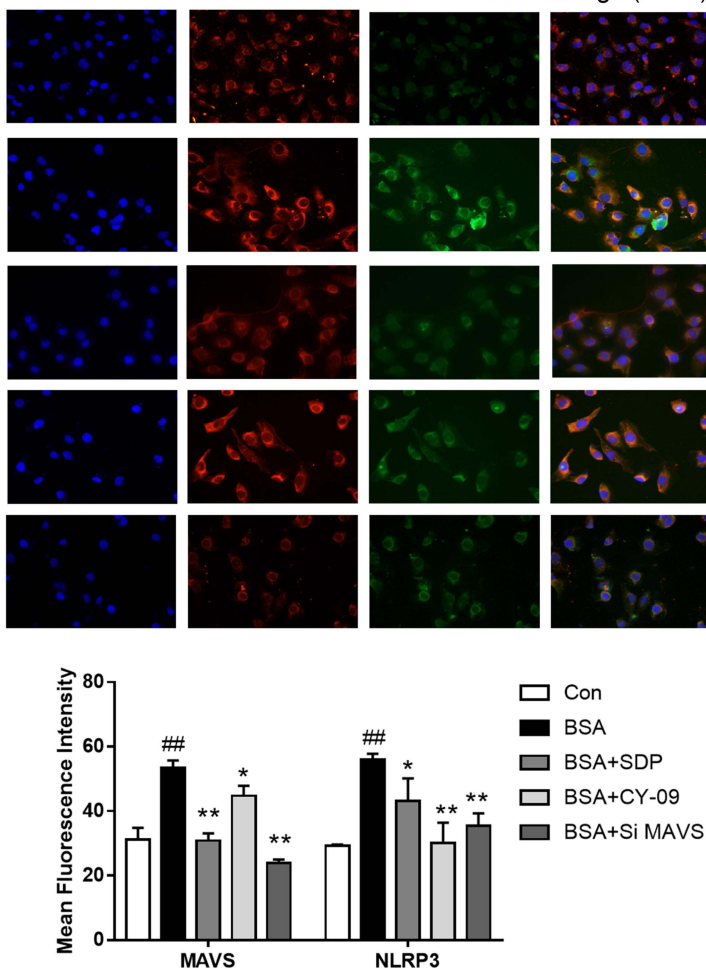

B
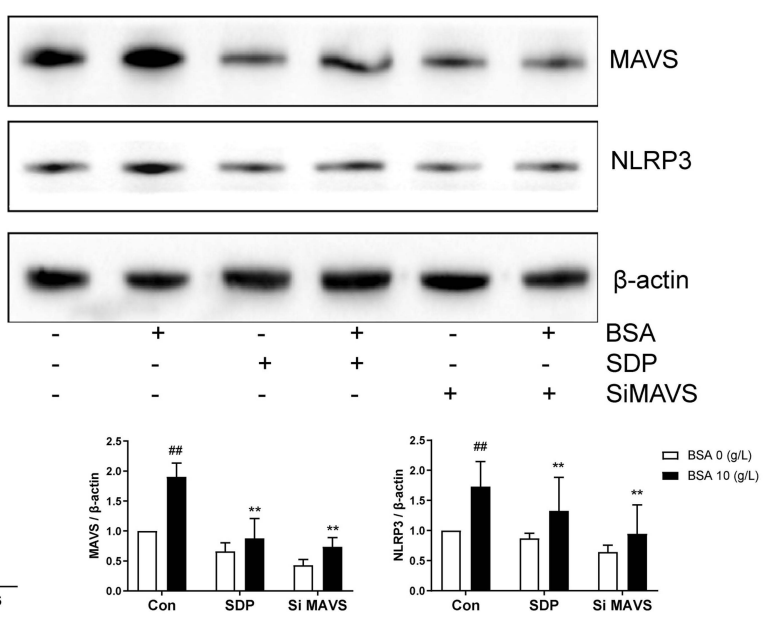

$\mathbf{E}$
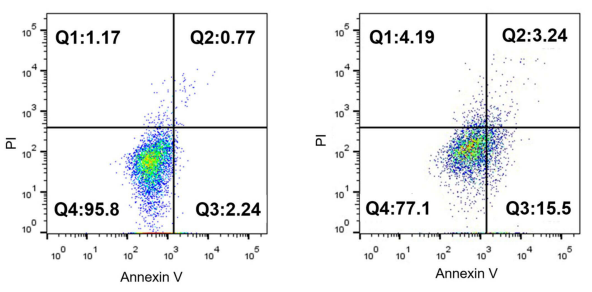

Con

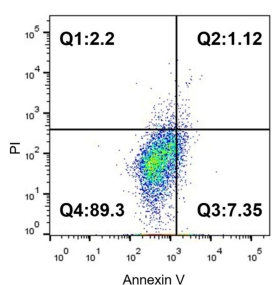

$B S A+S D P$
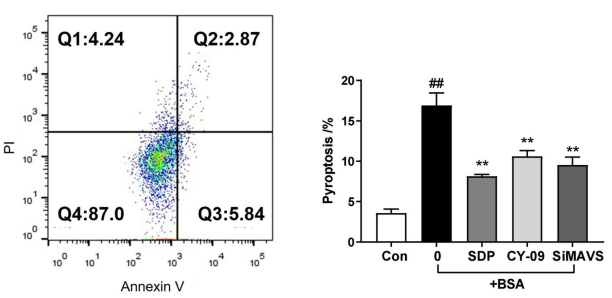

BSA+SiMAVS
C
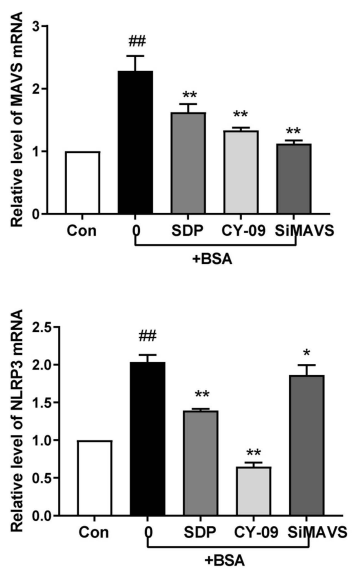

Figure 7 SDP inhibited the localization and activation of NLRP3 in mitochondria by regulating MAVS protein. (A, B) The effects of MAVS siRNA and SDP on BSA-induced cell injury. HK-2 cells were transfected with either NLRP3 siRNA or control siRNA for $8 \mathrm{~h}$. The transfected cells were incubated with BSA for $24 \mathrm{~h}$. HK-2 cells were divided into 6 groups - Control, BSA ( $10 \mathrm{~g} / \mathrm{L})$, SDP ( $\mathrm{g} / \mathrm{L})$, BSA ( $10 \mathrm{~g} / \mathrm{L})+\operatorname{SDP}(\mathrm{l} \mathrm{g} / \mathrm{L})$, siMAVS $(80 \mathrm{nmol} / \mathrm{L})$, and BSA ( $10 \mathrm{~g} / \mathrm{L})+\mathrm{MAVS}(80 \mathrm{nmol} / \mathrm{L})$. Cell lysates were subjected to Western blotting to measure MAVS and NLRP3 levels. (C) mRNA levels of NLRP3 and MAVS were measured using RT-PCR. (D) The co-localization of MAVS and NLRP3 was observed using immunofluorescence staining. (E) Annexin V -FITC and PI channels were used to observe cell pyroptosis. Data are expressed as means \pm SD; $n=3$. ${ }^{*} P<0.05$; ${ }^{\# \#} P<0.0$ l vs control. $* P<0.05 ; * * P<0.01$ vs the BSA group. 


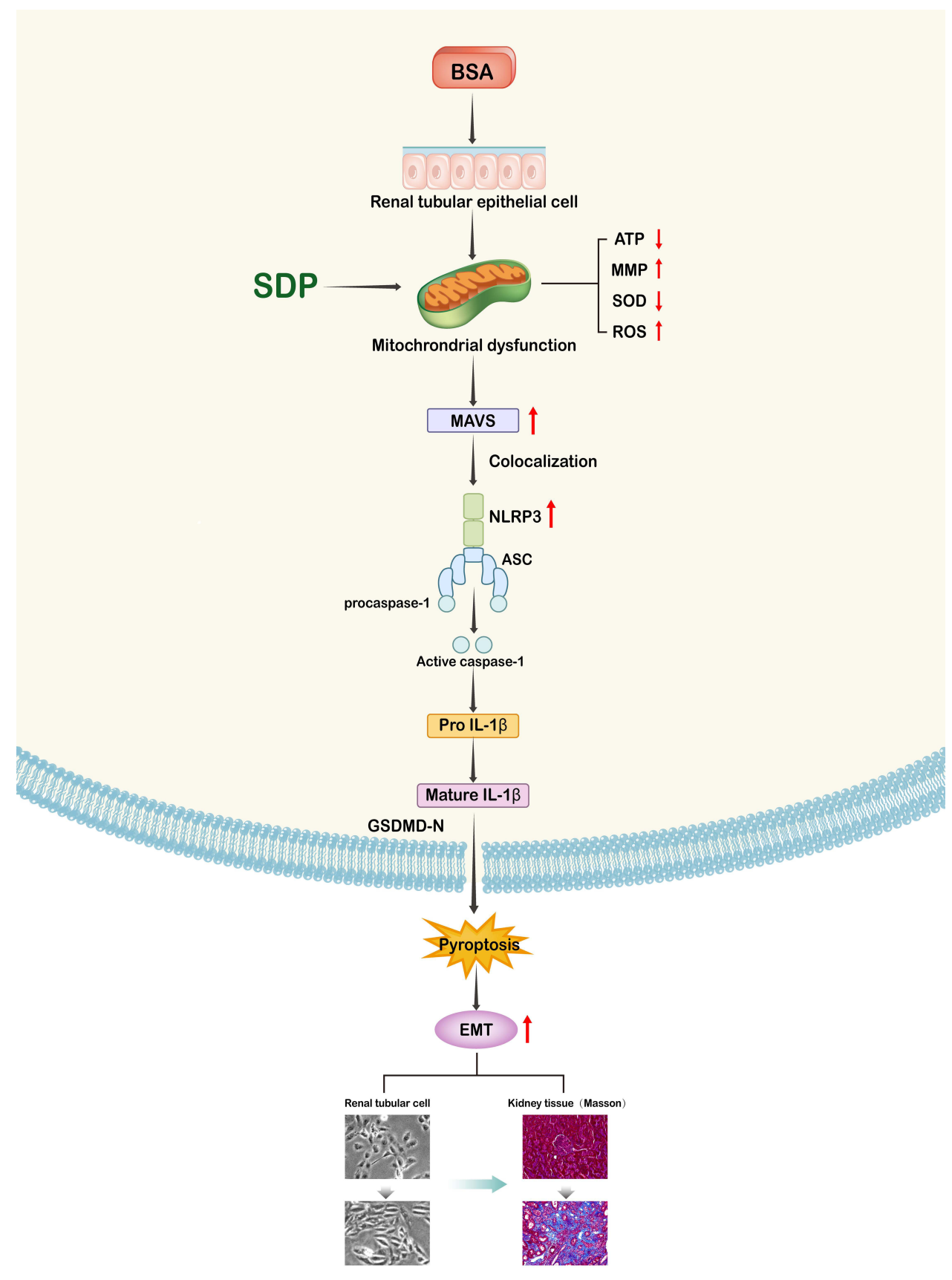

Figure 8 A schematic representation of the signaling pathways involved in the SDP-mediated inhibition of BSA in human renal tubular epithelial cells (HK-2 cells).

was knocked out. Compared to the BSA group, the siMAVS and SDP groups showed a reduced expression of MAVS, inhibition of NLRP3, and a decrease in the mRNA contents of MAVS and NLRP3 (Figure 7A-C). Immunofluorescence results suggested that MAVS colocated with mitochondria, and BSA enhanced NLRP3 colocalization with MAVS and increased the fluorescence.
SDP and siMAVS decreased their co-localization and fluorescence (Figure 7D). Interestingly, the CY-09 group showed decreased NLRP3 and MAVS fluorescence expression, suggesting that NLRP3 may regulate MAVS via positive feedback. Flow cytometry was performed to detect the positive rate of Annexin V and PI double-stained cells using channels PE and 488. Compared to the BSA 
group, SDP, CY-09, and siMAVS groups showed decreased pyroptosis as measured using flow cytometry (Figure 7E). These data suggested that SDP inhibits the activation of the NLRP3 inflammasome by regulating MAVS expression, thereby alleviating cell pyroptosis.

\section{Discussion}

Traditional Chinese medicine has been used in China for more than 2500 years. With developments in science and technology, several Chinese herbs and even their active constituents have been proved to be effective in the treatment of CKD. ${ }^{17-19}$ However, following the principle of compatibility of Traditional Chinese Medicine (TCM), the treatment effect of a combination is more obvious than that of individual drugs, and the clinical use of TCM is still dominated by the Chinese herbal compound prescription. $^{20,21}$ SDP has been used in the clinic for several years and has shown a satisfactory curative effect. It has been converted into granules (SDG) for convenience. The current study was the first to investigate the renal protective effect of SDP on adenine-induced renal failure rats. It was found that SDP not only improved renal function and fibrosis but also reduced proteinuria and inflammation. In vitro, the model of renal tubular epithelial cells established using albumin suggested that SDP might reduce pyroptosis by alleviating mitochondrial dysfunction, inhibiting the MAVS/NLRP3 inflammasome pathway, and further inhibiting the EMT of renal tubular epithelial cells (Figure 8).

Numerous clinical trials have shown that proteinuria is an independent predictor of CKD progression. The risk of ESKD and cardiovascular mortality were positively correlated with proteinuria. ${ }^{22}$ The protein in the initial urine is filtered through the glomerulus and reabsorbed from the renal tubules into the blood circulation system. Proteinuria may be caused due to immunologic and/or nonimmunologic factors that impair the glomerular filtration or damage the renal tubules. ${ }^{23}$ Proteinuria is a driving factor of renal tubulointerstitial inflammation and fibrosis. ${ }^{24}$ However, the exact mechanism underlying proteinuria is not clear. The animal model of renal injury was established by intragastric administration of adenine, and adenine was deposited into the renal tubules by the action of xanthine oxidase ${ }^{16}$ Compared to other rodent models of renal failure established using intravenous or surgical methods, intragastric administration of adenine helps not only strictly control drug dose and reduce mortality but also avoids inflammatory interference caused by external injury. Our data showed that after continuous administration of $2.5 \%$ adenine for 1 month and subsequent daily administration of $200 \mathrm{mg} / \mathrm{kg} / \mathrm{d}$ for 1 month, serum urea nitrogen, serum creatinine, serum uric acid, and serum cystatin $\mathrm{C}$ increased, and proteinuria was observed, consistent with clinical manifestations of CKD. Pathological staining and immunohistochemistry also confirmed the damage to renal tubules including atrophy, showing loss of structure, inflammation, and fibrosis of the renal interstitium. No serious glomerular injury was observed using pathological staining, and no obvious mesangial hyperplasia was observed in the glomerulus due to PAS staining, which led us to focus our attention on the renal tubules. Both SDG with a low dose $(5 \mathrm{~g} / \mathrm{kg} /$ day $)$ and a high dose $(10 \mathrm{~g} / \mathrm{kg} /$ day $)$ alleviated the injury, and the effect was more significant in the high-dose group.

The mechanism underlying proteinuria leading to renal tubulointerstitial damage is a field of active research. Currently known injury mechanisms include direct toxic effects of proteins, oxidative stress, chemical additives, inflammasome, apoptosis, cellular senescence, and autophagy. ${ }^{25}$ In our previous study, NLRP3 inflammasome was activated in response to proteinuria induced by doxorubicin-induced nephropathy. ${ }^{26}$ The assembly of NLRP3 inflammasome includes sensor-NLRP3, adapter-ACS, and progenase-procaspase- 1 . When stimulated by pathogenassociated molecular pattern (PAMP) and damageassociated molecular pattern (DAMP), the inflammasome acts as a polymeric protein complex to cause self-cleavage of caspase-1, resulting in the cleavage of IL-1 $\beta$ and IL-18 into mature IL-1 $\beta$ and IL-18, respectively, which are released by the cell and trigger inflammatory responses. Meanwhile, mature caspase-1 promotes Gasdermin D to form pores in the cell membrane and further enhances the release of mature IL-1 $\beta$ and IL-18. This process, called pyroptosis, can be induced by non-classical caspase- 4 , caspase-5, and caspase-11 in addition to classical caspase- $1 .{ }^{27,28}$ As a kind of programmed inflammatory necrotic cell death, moderate pyroptosis can maintain the balance of the internal environment but excessive pyroptosis leads to the release of a large number of proinflammatory factors and an inflammatory response. Currently, pyroptosis is known to be involved in the occurrence and development of infection, autoimmune diseases, neurodegenerative diseases, metabolic disorders, and other diseases. ${ }^{29}$ In the current study, the expression of NLRP3, Caspase-1, Gasdermin D, and IL-1 $\beta$ in the renal tissues and macrophage chemokines MCP-1, IL-1, IL-1 $\beta$, 
and IL-18 in the blood were measured. It was confirmed that adenine-induced renal injury was associated with a series of reactions induced by the NLRP3 inflammasome. Experimental evidence suggested that low and high doses of SDG attenuated these inflammatory responses but the underlying mechanism is not clear.

In addition to providing energy for cellular activities, mitochondria are also involved in regulating cellular functions, including the stabilization of calcium concentration in the internal environment, apoptosis, cell signal transduction, and senescence. ${ }^{30}$ Mitochondrial involvement in the regulation of immunity is an important discovery made in recent years. ${ }^{31}$ Evidence suggests that mitochondria activate the NLRP3 inflammasome by three pathways ${ }^{32}$ - (1) Mitochondria activate NLRP3 inflammasome by releasing ROS: When mitochondria become dysfunctional, they produce excess ROS, beyond their clearance limit, and excess ROS activate related signal transduction pathways, causing cell damage. Previous experiments have shown that a mitochondrial dysfunction is an early event in the podocyte and tubular epithelial cell injuries, and blocking mitochondrial ROS can reduce cell injury, suggesting that mitochondrial dysfunction plays an important role in the initial stages of cell injury. ${ }^{33,34}$ (2) Mitochondrial DNA activates NLRP3 inflammasome: mtDNA is the genetic material in mitochondria. When mitochondrial dysfunction occurs, mtDNA is released into the cytoplasm, becoming DAMP and binding to NLRP3. ${ }^{35}$ To further study the effect of mtDNA on the activation of the NLRP3 inflammasome, Nakahira et $\mathrm{al}^{36}$ overexpressed mtDNA in bone marrow-derived macrophages (BMDMs) cells and found that the overexpressed mtDNA induced the activation of NLRP3 inflammasome and the expression of IL-1 $\beta$ in a dose-dependent manner. This suggested that mtDNA released in the cytoplasm directly binds and activates NLRP3 inflammasome causing mitochondrial dysfunction. (3) Mitochondrial co-localization with NLRP3 is crucial for NLRP3 inflammasome activation: Toll-like receptors (TLRs) associated with cytoplasmic membranes and introns, as well as RIG-1, are recruited by mitochondrial outer membrane protein MAVS to initiate the type I interferon responses during viral infection. Initially, it was thought that the assembly of the activated NLRP3 inflammasome in the cytoplasm might not be recruited at the mitochondria. However, Subramanian et $\mathrm{al}^{37}$ demonstrated that NLRP3, in the resting state, is mainly located in the endoplasmic reticulum, and whereas NLRP3 and ASC are redistributed to the endoplasmic reticulum and mitochondria located in clusters around the nucleus by the NLRP3 inflammasome activator. Studies have further shown that MAVS protein is necessary for the optimal activity of the NLRP3 inflammasome. MAVS interacts with the N-terminus of NLRP3 during inflammasome activation. Therefore, MAVS not only mediates the virusresistant type I interferon response but also serves as a bridge for mitochondrial regulation of the NLRP3 inflammasome. ${ }^{38}$ In addition to MAVS, apoptosis inhibitor protein (c-FLIP), ${ }^{39}$ acetylated $\alpha$-tubulin, ${ }^{40}$ and cardiolipin ${ }^{41}$ on mitochondria also regulate NLRP3 mitochondrial localization.

In the current study, by measuring the mitochondrial function, it was observed that albumin affected the release of ATP, the level of mitochondrial membrane potential, the content of SOD, and the release of ROS, indicating that albumin can cause mitochondrial function in HK-2 cells, and SDP can alleviate the dysfunction. In the past, MAVS protein was considered to activate the immune-related signaling pathway and induce the expression of interferons to participate in the antiviral immune response. ${ }^{37}$ It has also been reported that MAVS regulated NLRP3 by repositioning NLRP3 in early hypoxia. Evidence suggested that MAVS protein expressed on the mitochondria is stimulated by albumin to increase its expression. Meanwhile, the co-localization of NLRP3 and MAVS is enhanced along with an increase in their expression. When siRNA was used to knock down MAVS, the co-localization and expression of MAVS and NLRP3 weakened, and similar results are found in the SDP group. It is worth noting that CY-09, an inhibitor of NLRP3, also inhibited the MAVS protein. Rather than a direct causal relationship, a complex interaction between NLRP3 and CY-09 is speculated.

\section{Conclusion}

In conclusion, the study showed that SDP improved proteinuria and renal fibrosis, reduced inflammation, and protected renal function in an adenine-induced renal injury rat model. In addition, we explored the regulation of NLRP3 by MAVS induced by mitochondrial dysfunction, which is involved in inflammation-induced cell pyroptosis and renal tubular epithelial EMT, and may be a novel target for the treatment of CKD. Our results indicated that SDP could protect renal tubular epithelial cells from cell pyroptosis and EMT by regulating the albumin-induced mitochondrial dysfunction/ MAVS/ NLRP3-ASC-caspase-1 inflammasome signaling pathway.

\section{Acknowledgments}

This work was supported by the National Natural Science Foundation of China (81873270), the Science Project of Jiangsu Province Hospital of Traditional Chinese Medicine (Y2018RC17), the Top Talents Training Program of Jiangsu 
Province (WSN-012), the Advantage Discipline Project of Nanjing University of Chinese Medicine (ZYX03KF076), and the Medical International Cooperation Project of Nanjing Municipal Science and Technology Bureau (202002051).

\section{Disclosure}

The authors report no conflicts of interest.

\section{References}

1. Jha V, Garcia-Garcia G, Iseki K, et al. Chronic kidney disease: global dimension and perspectives. Lancet. 2013;382(9888):260-272.

2. Stevens PE, Levin A. Evaluation and management of chronic kidney disease: synopsis of the kidney disease: improving global outcomes 2012 clinical practice guideline. Ann Intern Med. 2013;158 (11):825-830.

3. Obrador GT, Levin A, Hotspots: CKD. Challenges and areas of opportunity. Semin Nephrol. 2019;39(3):308-314.

4. Nastase MV, Zeng-Brouwers J, Wygrecka M, et al. Targeting renal fibrosis: mechanisms and drug delivery systems. Adv Drug Deliv Rev. 2018;129:295-307.

5. Lovisa S, LeBleu VS, Tampe B, et al. Epithelial-to-mesenchymal transition induces cell cycle arrest and parenchymal damage in renal fibrosis. Nat Med. 2015;21(9):998-1009.

6. Carew RM, Wang B, Kantharidis P. The role of EMT in renal fibrosis. Cell Tissue Res. 2012;347(1):103-116.

7. Sheng L, Zhuang S. New insights into the role and mechanism of partial epithelial-mesenchymal transition in kidney fibrosis. Front Physiol. 2020;11:569322.

8. Mangan MSJ, Olhava EJ, Roush WR, et al. Targeting the NLRP3 inflammasome in inflammatory diseases. Nat Rev Drug Discov. 2018;17(8):588-606.

9. Haneklaus M, O'Neill LA. NLRP3 at the interface of metabolism and inflammation. Immunol Rev. 2015;265(1):53-62.

10. Cuevas S, Pelegrín P. Pyroptosis and redox balance in kidney diseases. Antioxid Redox Signal. 2021;35(1):40-60.

11. Kim SM, Kim YG, Kim DJ, et al. Inflammasome-independent role of NLRP3 mediates mitochondrial regulation in renal injury. Front Immunol. 2018;9:2563.

12. Kong Y, Feng W, Zhao X, et al. Statins ameliorate cholesterol-induced inflammation and improve AQP2 expression by inhibiting NLRP3 activation in the kidney. Theranostics. 2020;10 (23):10415-10433.

13. Chen H, Fang Y, Wu J, et al. RIPK3-MLKL-mediated necroinflammation contributes to AKI progression to CKD. Cell Death Dis. 2018;9(9):878.

14. Anders HJ, Suarez-Alvarez B, Grigorescu M, et al. The macrophage phenotype and inflammasome component NLRP3 contributes to nephrocalcinosis-related chronic kidney disease independent from IL-1-mediated tissue injury. Kidney Int. 2018;93(3):656-669.

15. Zhu YY, Zhou EC, Gao K, et al. Protective effect and mechanism of aqueous extract of Perillae Folium on Adriamycin-induced oxidative injury in HK-2 Cell. Chinese J Exp Trad Med Formulae. 2019:50-57. doi:10.13422/j.cnki.syfjx.20191238

16. Diwan V, Brown L, Gobe GC. Adenine-induced chronic kidney disease in rats. Nephrology (Carlton). 2018;23(1):5-11.

17. Zhang HW, Lin ZX, Tung YS, et al. Cordyceps sinensis (a traditional Chinese medicine) for treating chronic kidney disease. Cochrane Database Syst Rev. 2014;12:CD008353.
18. Zhong Y, Menon MC, Deng Y, Chen Y, He JC. Recent advances in traditional Chinese medicine for kidney disease. Am J Kidney Dis. 2015;66(3):513-522.

19. Qu S, Dai C, Hao Z, et al. Chlorogenic acid prevents vancomycin-induced nephrotoxicity without compromising vancomycin antibacterial properties. Phytother Res. 2020;34(12):3189-3199.

20. Qin T, Wu L, Hua Q, et al. Prediction of the mechanisms of action of Shenkang in chronic kidney disease: a network pharmacology study and experimental validation. J Ethnopharmacol. 2020;246:112-128.

21. Wang X, Yu S, Jia Q, et al. NiaoDuQing granules relieve chronic kidney disease symptoms by decreasing renal fibrosis and anemia. Oncotarget. 2017;8(34):55920-55937.

22. Sarnak MJ, Astor BC. Implications of proteinuria: CKD progression and cardiovascular outcomes. Adv Chronic Kidney Dis. 2011;18 (4):258-266.

23. Taal MW, Brenner BM. Renal risk scores: progress and prospects. Kidney Int. 2008;73(11):1216-1219.

24. Strutz FM. EMT and proteinuria as progression factors. Kidney Int. 2009;75(5):475-481.

25. Keane WF. Proteinuria: its clinical importance and role in progressive renal disease. Am J Kidney Dis. 2000;35(4 Suppl 1):S97-S105.

26. Li W, He W, Xia P, et al. Total extracts of abelmoschus manihot L. attenuates adriamycin-induced renal tubule injury via suppression of ROS-ERK1/2-mediated NLRP3 inflammasome activation. Front Pharmacol. 2019;10:567.

27. Mangan MSJ, Olhava EJ, Roush WR, et al. Targeting the NLRP3 inflammasome in inflammatory diseases [published correction appears in Nat Rev Drug Discov . 2018 Sep;17(9):688]. Nat Rev Drug Discov. 2018;17(8):588-606.

28. Zhang WJ, Chen SJ, Zhou SC, Wu SZ, Wang H. Inflammasomes and Fibrosis. Front Immunol. 2021;12:643149.

29. Van Opdenbosch N, Lamkanfi M. Caspases in cell death, inflammation, and disease. Immunity. 2019;50(6):1352-1364.

30. Nunnari J, Suomalainen A. Mitochondria: in sickness and in health. Cell. 2012;148(6):1145-1159.

31. Moehlman AT, Youle RJ. Mitochondrial quality control and restraining innate immunity. Annu Rev Cell Dev Biol. 2020;36:265-289.

32. Zhou R, Yazdi AS, Menu P, Tschopp J. A role for mitochondria in NLRP3 inflammasome activation [published correction appears in Nature. 2011 Jul 7;475(7354):122]. Nature. 2011;469(7329):221-225.

33. Pi S, Nie G, Wei Z, et al. Inhibition of ROS/NLRP3/Caspase-1 mediated pyroptosis alleviates excess molybdenum-induced apoptosis in duck renal tubular epithelial cells. Ecotoxicol Environ Saf. 2021;208:111528.

34. Yan J, Li Y, Yang H, et al. Interleukin-17A participates in podocyte injury by inducing IL-1 $\beta$ secretion through ROS-NLRP3 inflammasome-caspase-1 pathway. Scand J Immunol. 2018;87(4):e12645.

35. Zhong Z, Liang S, Sanchez-Lopez E, et al. New mitochondrial DNA synthesis enables NLRP3 inflammasome activation. Nature. 2018;560(7717):198-203.

36. Nakahira K, Haspel JA, Rathinam VA, et al. Autophagy proteins regulate innate immune responses by inhibiting the release of mitochondrial DNA mediated by the NALP3 inflammasome. Nat Immunol. 2011;12(3):222-230.

37. Subramanian N, Natarajan K, Clatworthy MR, et al. The adaptor MAVS promotes NLRP3 mitochondrial localization and inflammasome activation. Cell. 2013;153(2):348-361.

38. Mills EL, Kelly B, O’Neill LAJ. Mitochondria are the powerhouses of immunity. Nat Immunol. 2017;18(5):488-498.

39. Wu YH, Kuo WC, Wu YJ, et al. Participation of c-FLIP in NLRP3 and AIM2 inflammasome activation. Cell Death Differ. 2014;21 (3):451-461. 
40. Qiao S, Lv C, Tao Y, et al. Arctigenin disrupts NLRP3 inflammasome assembly in colonic macrophages via downregulating fatty acid oxidation to prevent colitis-associated cancer. Cancer Lett. 2020;491:162-179.
41. Iyer SS, He Q, Janczy JR, et al. Mitochondrial cardiolipin is required for Nlrp3 inflammasome activation. Immunity. 2013;39(2):311-323.

\section{Publish your work in this journal}

The Journal of Inflammation Research is an international, peerreviewed open-access journal that welcomes laboratory and clinical findings on the molecular basis, cell biology and pharmacology of inflammation including original research, reviews, symposium reports, hypothesis formation and commentaries on: acute/chronic inflammation; mediators of inflammation; cellular processes; molecular mechanisms; pharmacology and novel anti-inflammatory drugs; clinical conditions involving inflammation. The manuscript management system is completely online and includes a very quick and fair peerreview system. Visit http://www.dovepress.com/testimonials.php to read real quotes from published authors. 